\title{
Analysis of a Temperature- and Rainfall-dependent Model for Malaria Transmission Dynamics
}

\author{
Kamaldeen Okuneye and Abba B. Gumel* \\ School of Mathematical and Statistical Sciences, Arizona State University, Tempe, Arizona, USA.
}

March 16, 2016

\begin{abstract}
A new non-autonomous model is designed and used to assess the impact of variability in temperature and rainfall on the transmission dynamics of malaria in a population. In addition to adding age-structure in the host population and the dynamics of of immature malaria mosquitoes, a notable feature of the new model is that recovered individuals do not revert to wholly-susceptible class (that is, recovered individuals enjoy reduced susceptibility to new malaria infection). In the absence of disease-induced mortality, the disease-free solution of the model is shown to be globally-asymptotically stable when the associated reproduction ratio is less than unity. The model has at least one positive periodic solution when the reproduction ratio exceeds unity (and the disease persists in the community in this case). Detailed uncertainty and sensitivity analysis, using mean monthly temperature and rainfall data from KwaZulu-Natal province of South Africa, shows that the top three parameters of the model that have the most influence on the disease transmission dynamics are the mosquito carrying capacity, transmission probability per contact for susceptible mosquitoes and human recovery rate. Numerical simulations of the model show that, for the KwaZulu-Natal province, malaria burden increases with increasing mean monthly temperature and rainfall in the ranges $\left([17-25]^{\circ} \mathrm{C}\right.$ and $[32-110] \mathrm{mm}$ ), respectively (and decreases with decreasing mean monthly temperature and rainfall values). In particular, transmission is maximized for mean monthly temperature and rainfall in the ranges $[21-25]^{\circ} \mathrm{C}$ and $[95-125] \mathrm{mm}$. This occurs for a six-month period in KwaZulu-Natal (hence, this study suggests that anti-malaria control efforts should be intensified during this period). It is shown, for the fixed mean monthly temperature of KwaZulu-Natal, that malaria burden decreases whenever the amount of rainfall exceeds a certain threshold value. It is further shown (through sensitivity analysis and numerical simulations) that incorporating host age-structure and reduced susceptibility due to prior malaria infection has marginal effect on the transmission dynamics of the disease.
\end{abstract}

Key words: age-structure; malaria; non-autonomous model; stability; reproduction ratio.

*Corresponding author: A.B. Gumel (Email: agumel@asu.edu) 


\section{Introduction}

A number of recent studies have established the significant direct role climate variables, such as temperature and rainfall, play on the transmission dynamics of vector-borne diseases (VBDs) $[1,29,32,42,49,51,52,54,57,59,67,74,75]$. Furthermore, changing wind patterns has been shown to have significant impact on the dynamics of some vectors [25]. Malaria, being the most important VBD [27], receives the most attention (half the world's population are at risk of malaria infection. The disease, which is spread in humans following an effective bite by an infected adult female Anopheles mosquito (the malaria vector), accounts for 250 million infections and approximately one million mortality annually, with $85 \%$ of the mortality occurring in children under the age of five [27, 73]).

The specific role temperature plays on the dynamics of malaria takes many different forms. For example, increases in temperature generally causes the malaria vector to feed more frequently [26]. Furthermore, the average lifespan of the vector decreases rapidly (from the average 21 days) for higher temperatures (beyond $[30-32]^{\circ} \mathrm{C}$ ) [20]. The temperature sensitivity of malaria parasites to mosquito hosts has long been established $[9,15,38]$. Recent studies have shown that the maturation rate of the malaria parasite (Plasmodium) inside the host significantly decreases with increasing temperature (for example, as noted by [38], the maturation rate decreases from 19 days at $22^{\circ} \mathrm{C}$ to 8 days at $30^{\circ} \mathrm{C}$ ) [20]. Warmer temperature (warmer waters) also leads to faster maturation of the mosquito larvae [26]. Mosquito survival is greatly impacted at excessive temperatures [22]. Finally, in addition to its direct effects on vector and parasite development, temperature can also have a profound effect on vector physiology and immune responses [46, 47, 66].

The effect of rainfall on malaria transmission dynamics is directly tied to the availability of mosquito breeding sites (the use of precipitation as an empirical predictor of incidence, in addition to its direct impact on mosquito abundance, is well established [54]). Although total rainfall generally increases the availability and productivity of mosquito breeding sites (immature stages of the mosquito are aquatic, hence increasing rainfall increases breeding habitats and, consequently, mosquito abundance) [40], excessive rainfall can lead to washout effect of the breeding sites [40]. Hence, the timing, frequency and quantity of precipitation are important, and likely differ for different mosquito species [54]. Furthermore, as noted by Parham et al. [54], total rainfall is expected to have a highly nonlinear effect on mosquito production (in particular, having qualitatively different effects on container-breeding versus seasonal or permanent wetland breeding species).

Although climatic variability, especially changes in temperature and rainfall, influences the dynamics of malaria and other VBDs, this influence may be affected by non-climatic factors, such as epidemiological, environmental, socio-economic and demographic factors (see [54] and some of the references therein). Hence, as noted in [54], quantifying the impact of climate change on the dynamics of VBDs requires a better understanding of the role these non-climatic factors (and their combinations) have on the overall vector-pathogenhost dynamics. The purpose of the current study is to model the population-level effect of variabilities in temperature and rainfall patterns on the transmission dynamics of malaria.

Numerous mathematical models have been designed and used to quantify the impact of climate variables on the transmission dynamics of VBDs. These models fall in two main categories, namely mechanistic or process-based models (which represent the dynamics of the 
disease using differential equations) and statistical models (which are typically based on the use of regression models adapted to time-series data to describe the correlation or relationship between VBDs and environmental indicators of climate, climate change, meteorological factors and extreme weather events $[2,3,7,10,14,54,58,62])$.

Using a semi-parametric model, Egbendewe-Mondzozo et al. [20] showed that a marginal increase in temperature and precipitation could cause a significant change in malaria cases in many African countries. Blanford et al. [5] modeled the role of diurnal temperature range and mean temperature on the extrinsic incubation period of the malaria parasite. Lunde et al. [37] compared temperature-dependent mortality models for Anopheless gambiae sensu stricto. Mordecai et al. [45] showed, using a non-linear thermal response model, that the optimal temperature for malaria transmission is $25^{\circ} \mathrm{C}$ and decreases significantly beyond $28^{\circ} \mathrm{C}$. Using a mechanistic repeated exposure malaria model, which also explicitly modeled the aquatic stages of the malaria vector, Agusto et al. [1] showed that the suitable temperature for mosquito growth (hence, malaria transmission) in 67 cities in sub-Saharan Africa lies in the range $[16-28]^{\circ} \mathrm{C}$. A delay differential equation temperature-dependent malaria model was developed by Beck-Johnson et al. [4]. Using a mechanistic model which incorporates temperature, rainfall and other environmental variables (such as wind speed and relative humidity), Parham et al. [53] showed that these abiotic factors play significant roles in vector abundance.

As noted by Parham et al. [53], although statistical models have been useful in providing insight into the correlation between environmental variables and transmission intensity, the fundamental importance of mechanistic (process-based) models cannot be over-emphasized. For instance, unlike in the case of statistical models, mechanistic models allow for deeper understanding of the role of internal (due to biological processes) versus external (such as those due to changes in environmental variables) drivers of transmission (these biological processes, which drive the malaria transmission dynamics, are coupled within a dynamicallychanging environment over a range a timescales [53]). The overwhelming majority of the VBD mechanistic modelling work that incorporate environmental variables published so far in the investigated the impact of variability in temperature only. To the authors' knowledge, the model in [53] is the only mechanistic modeling study to combine the effect of temperature and rainfall on malaria transmission dynamics. Consequently, the objective of the current study is to extend the work in [53] by designing a new temperature and rainfall-dependent mechanistic malaria model that incorporates some more pertinent climatic and non-climatic features and factors not considered in [53] (such as host age-structure, dynamics of immature mosquitoes, reduced susceptibility due to prior malaria infection etc.). The model to be developed in this paper is formulated in Section 2. The autonomous version of the model is analysed in Section 3. The full non-autonomous model is analysed and simulated in Section 4. Uncertainty and sensitivity analyses are carried out on the parameters of the model in Section 5. Numerical simulation results are also reported.

\section{Model Formulation}

The total human population at time $t$, denoted by $N_{h}(t)$, is split into sub-populations of children (typically under the age of 17) and adults. The total sub-population of children 
at time $t$, denoted by $N_{c}(t)$, consists of children who are wholly susceptible $\left(S_{c}(t)\right)$, susceptible with some immunity due to recovery from prior malaria infection $\left(S_{c r}(t)\right)$, exposed $\left(E_{c}(t)\right)$, exposed with prior immunity $\left(E_{c r}(t)\right)$, infectious $\left(I_{c}(t)\right)$, infectious with prior immunity $\left(I_{c r}(t)\right)$, recovered $\left(W_{c}(t)\right)$ and recovered with prior immunity $\left(W_{c r}(t)\right)$. Similarly, the total sub-population of adults at time $t$, denoted by $N_{a}(t)$, is split into adults who are wholly susceptible $\left(S_{a}(t)\right)$, susceptible with some immunity due to recovery from prior malaria infection $\left(S_{a r}(t)\right)$, exposed $\left(E_{a}(t)\right)$, exposed with prior immunity $\left(E_{a r}(t)\right)$, infectious $\left(I_{a}(t)\right)$, infectious with prior immunity $\left(I_{a r}(t)\right)$, recovered $\left(W_{a}(t)\right)$ and recovered with prior immunity $\left(W_{a r}(t)\right)$. Thus, $N_{h}(t)=N_{c}(t)+N_{a}(t)$, where:

$$
\begin{aligned}
& N_{c}(t)=S_{c}(t)+S_{c r}(t)+E_{c}(t)+E_{c r}(t)+I_{c}(t)+I_{c r}(t)+W_{c}(t)+W_{c r}(t), \\
& N_{a}(t)=S_{a}(t)+S_{a r}(t)+E_{a}(t)+E_{a r}(t)+I_{a}(t)+I_{a r}(t)+W_{a}(t)+W_{a r}(t) .
\end{aligned}
$$

The total mosquito population at time $t$, denoted by $N_{v}(t)$, is sub-divided into sub-populations of immature mosquitoes (egg, larva and pupa stages), denoted by $\left(M_{a}(t)\right)$, and adult mosquitoes (denoted by $N_{m}(t)$ ), so that

$$
N_{v}(t)=M_{a}(t)+N_{m}(t),
$$

where $N_{m}(t)$ as the sum of uninfected adult mosquitoes $\left(M_{s}(t)\right)$ and infected adult mosquitoes $\left(M_{i}(t)\right)$ (i.e., $N_{m}(t)=M_{s}(t)+M_{i}(t)$ ).

The non-autonomous age-structured model for malaria transmission dynamics in a population is given by the following deterministic system of non-linear differential equations (a flow diagram of the model is depicted in Figure 1; the state variables and parameters of the 
model are described in Table 1):

$$
\begin{aligned}
& \frac{d S_{c}(t)}{d t}=\Pi_{c}-\lambda_{c}(T) S_{c}-\left(\xi_{h}+\mu_{h}\right) S_{c}, \\
& \frac{d S_{c r}(t)}{d t}=\psi_{c}\left(W_{c}+\theta_{c r} W_{c r}\right)-\left(1-\epsilon_{c}\right) \lambda_{c}(T) S_{c r}-\left(\xi_{h}+\mu_{h}\right) S_{c r}, \\
& \frac{d E_{c}(t)}{d t}=\lambda_{c}(T) S_{c}-\left(\sigma_{c}+\mu_{h}\right) E_{c}, \\
& \frac{d E_{c r}(t)}{d t}=\left(1-\epsilon_{c}\right) \lambda_{c}(T) S_{c r}-\left(\sigma_{c r}+\mu_{h}\right) E_{c r}, \\
& \frac{d I_{c}(t)}{d t}=\sigma_{c} E_{c}-\left(\gamma_{c}+\mu_{h}+\delta_{c}\right) I_{c}, \\
& \frac{d I_{c r}(t)}{d t}=\sigma_{c r} E_{c r}-\left(\gamma_{c r}+\mu_{h}+\delta_{c r}\right) I_{c r} \text {, } \\
& \frac{d W_{c}(t)}{d t}=\gamma_{c} I_{c}-\left(\mu_{h}+\psi_{c}\right) W_{c}, \\
& \frac{d W_{c r}(t)}{d t}=\gamma_{c r} I_{c r}-\left(\mu_{h}+\theta_{c r} \psi_{c}\right) W_{c r}, \\
& \frac{d S_{a}(t)}{d t}=\xi_{h} S_{c}-\lambda_{a}(T) S_{a}-\mu_{h} S_{a}, \\
& \frac{d S_{a r}(t)}{d t}=\psi_{a}\left(W_{a}+\theta_{a r} W_{a r}\right)+\xi_{h} S_{c r}-\left(1-\epsilon_{a}\right) \lambda_{a}(T) S_{a r}-\mu_{h} S_{a r}, \\
& \frac{d E_{a}(t)}{d t}=\lambda_{a}(T) S_{a}-\left(\sigma_{a}+\mu_{h}\right) E_{a}, \\
& \frac{d E_{a r}(t)}{d t}=\left(1-\epsilon_{a}\right) \lambda_{a}(T) S_{a r}-\left(\sigma_{a r}+\mu_{h}\right) E_{a r}, \\
& \frac{d I_{a}(t)}{d t}=\sigma_{a} E_{a}-\left(\gamma_{a}+\mu_{h}+\delta_{a}\right) I_{a} \\
& \frac{d I_{a r}(t)}{d t}=\sigma_{a r} E_{a r}-\left(\gamma_{a r}+\mu_{h}+\delta_{a r}\right) I_{a r}, \\
& \frac{d W_{a}(t)}{d t}=\gamma_{a} I_{a}-\left(\mu_{h}+\psi_{a}\right) W_{a} \\
& \frac{d W_{a r}(t)}{d t}=\gamma_{a r} I_{a r}-\left(\mu_{h}+\theta_{a r} \psi_{a}\right) W_{a r}, \\
& \frac{d M_{a}(t)}{d t}=\alpha_{l}(T)\left(1-\frac{M_{a}}{K_{v}}\right)\left(M_{s}+M_{i}\right)-\xi_{l}(T, R) M_{a}-\mu_{l}(T) M_{a} \\
& \frac{d M_{s}(t)}{d t}=\xi_{l}(T, R) M_{a}-\lambda_{m}(T) M_{s}-\mu_{m}(T) M_{s}, \\
& \frac{d M_{i}(t)}{d t}=\lambda_{m}(T) M_{s}-\mu_{m}(T) M_{i},
\end{aligned}
$$

where the infection rates for children $\left(\lambda_{c}(T)\right)$, adults $\left(\lambda_{a}(T)\right)$ and vectors $\left(\lambda_{m}(T)\right)$ are given, respectively, by:

$$
\lambda_{c}(T)=\frac{b_{m}(T) \beta_{c} M_{i}}{N_{h}}, \lambda_{a}(T)=\frac{b_{m}(T) \beta_{a} M_{i}}{N_{h}}, \lambda_{m}(T)=\frac{b_{m}(T) \beta_{m}\left[I_{a}+I_{c}+\eta\left(I_{a r}+I_{c r}\right)\right]}{N_{h}} .
$$


In (2.1) and (2.2), $T=T(t)$ and $R=R(t)$ denote temperature and rainfall at time $t$ (each of these functions is assumed to be continuous, bounded, positive and $\tau$-periodic). In (2.1), $\Pi_{c}$ is the recruitment rate for children (by birth or immigration), $\lambda_{c}(T)$ is the infection rate of susceptible children from infectious mosquitoes (where $b_{m}(T)$ is the temperature-dependent per capital biting rate of mosquitoes and $\beta_{i}(i=c, a, m)$ is the probability of infection per bite), $\mu_{c}$ is the natural death rate, $\xi_{h}$ is the maturation rate of children into adulthood. Furthermore, $\psi_{c}$ is the rate at which recovered children move to the $S_{c r}$ class (of partiallyimmuned susceptible children), $0<\theta_{c r}<1$ is the modification parameter that measures the relative increase in immunity among the individuals after re-infection. The parameter $0<\epsilon_{c}<1$ represents the assumed reduction in infectivity of partially-immuned susceptible individuals (in relation to wholly-susceptible children). The parameter $\sigma_{c}\left(\sigma_{c r}\right)$ measures the rate at which children in the $E_{c}\left(E_{c r}\right)$ class develop clinical symptoms of malaria while the parameter $\gamma_{c}\left(\gamma_{c r}\right)$ measures the rate of recovery of recovery of symptomatic children from the $I_{c}\left(I_{c r}\right)$. The equations for the adult population are similarly derived (see also Table 1 for the description of the parameters of the model (2.1)).

Immature mosquitoes (eggs, larvae and pupae) are lumped into a single compartment $\left(M_{a}(t)\right)$ for computational convenience (see also $\left.[1,28,29,30]\right)$. The population of immature mosquitoes is generated at the temperature-dependent rate $\alpha_{l}(T)\left(1-\frac{M_{a}}{K_{v}}\right)$, where $\alpha_{l}(T)$ is the temperature-dependent egg deposition rate. Following [1], it is assumed that the population of immature mosquitoes is limited by a carrying capacity $K_{v}$ (where $K_{v}>M_{a}(t)$ for all $t$ depends on the available nutrients and breeding space $[28,29,30])$. Hence, the term $\alpha_{l}(T)\left(1-\frac{M_{a}}{K_{v}}\right)$ represents the logistic growth rate of immature mosquitoes. The population of immature mosquitoes is decreased by maturation to adult mosquitoes (at a temperatureand rainfall-dependent rate $\xi_{l}(T, R)$ ) and natural death (at a temperature-dependent rate $\left.\mu_{l}(T)\right)$. Susceptible adult mosquitoes acquire malaria infection at the rate $\lambda_{m}(T)$. Adult mosquitoes suffer natural death at a temperature-dependent rate $\mu_{m}(T)$. Although rainfall has been shown to generally increase malaria prevalence [65], it has also been reported that excessive rainfall can reduce transmission, due to the flushing out of aquatic breeding habitats [31]. It should be mentioned that, in this study (unlike in [1]), ambience and water temperature are assumed to be approximately equal (see $[1,49,55,56]$ for discussion on the relations between the two temperatures).

\subsection{Temperature and Rainfall-dependent Parameters}

The temperature- and rainfall-dependent parameters of the model (2.1) are defined as follows. The mosquito biting rate $b_{m}(T)$, defined using the quadratic function used in [45], is given by (see Figure 2A)

$$
b_{m}(T)=-0.00014 T^{2}+0.027 T-0.322 .
$$

The per-capita mosquito egg deposition rate, $\alpha_{l}(T)$, is given by (Figure 2B) [45]

$$
\alpha_{l}(T)=-0.153 T^{2}+8.61 T-97.7 \text {. }
$$


Similarly, following [45], the per-capita death rate of the adult mosquitoes, $\mu_{m}(T)$, is defined as (Figure 2C)

$$
\mu_{m}(T)=-\ln \left(-0.000828 T^{2}+0.0367 T+0.522\right) .
$$

Furthermore, following [53], the per-capita death rate of the immature mosquitoes, $\mu_{l}(T)$, is defined as (Figure 2D)

$$
\mu_{l}(T)=\frac{1}{8.560+20.654\left[1+\left(\frac{T}{19.759}\right)^{6.827}\right]^{-1}} .
$$

It is worth noting that the effect of rainfall on the death rate of immature mosquitoes, due, for instance, to flushing out effect for excessive rainfall, is incorporated in the maturation parameter, $\xi_{l}(T, R)$, below. Finally, following [45, 52], the temperature- and rainfall-dependent maturation rate of immature mosquitoes, $\xi_{l}(T, R)$, is defined as (Figure $2 \mathrm{E}$ )

$$
\xi_{l}(T, R)=\frac{B(T) p_{E}(R) p_{L}(T, R) p_{P}(R)}{\tau_{E A}(T)},
$$

where, $B(T)$ is the lifetime number of eggs laid by adult mosquitoes (in other words, $B(T)$ is defined as the total number of eggs laid per day $(E P D(T))$ by a mosquito multiplied by the average adult lifespan $\left.\left(1 / \mu_{m}(T)\right)\right)$ [45]. That is,

$$
B(T)=\frac{E F D(T)}{\mu_{m}(T)}
$$

with [45],

$$
E F D(T)=-0.153 T^{2}+8.61 T-97.7 \text {. }
$$

The parameters $p_{E}(R), p_{L}(T, R)$ and $p_{P}(R)$ are the daily survival probabilities of eggs, larvae and pupae, respectively. It is assumed that temperature and rainfall act independently on the survival probability of larvae [19], such that $p_{L}(T, R)=p_{L}(T) p_{L}(R)$. Following [52] (Supplemental Material), the rainfall-dependent daily probability of survival of immature mosquitoes, $p_{i}(R)$ (with $i=\{E, L, P\}$ ) is given by

$$
p_{i}(R)=\left(4 p_{M i} / R_{L}^{2}\right) R\left(R_{L}-R\right), i=\{E, L, P\},
$$

where $p_{M i}$ is the peak daily survival probability of immature mosquito stage $i(i=E=$ eggs; $i=L=$ larvae; $i=P=$ pupae) and $R_{L}>R(t)>0$, for all $t$, is the maximum rainfall threshold in the community. The temperature-dependent daily probability of survival of larvae, denoted by $p_{L}(T)$, is given in [52] (Supplemental Material) as

$$
p_{L}(T)=e^{-(0.00554 T-0.06737)} .
$$

Hence, using (2.6), with $i=L$, and (2.7), it follows that

$$
p_{L}(T, R)=\left(4 p_{M L} / R_{L}^{2}\right) R\left(R_{L}-R\right) \times e^{-(0.00554 T-0.06737)} .
$$


The total development time, from egg to adult mosquito, denoted by $\tau_{E A}(T)$, is given by [45]

$$
\tau_{E A}(T)=\frac{1}{-0.00094 T^{2}+0.049 T-0.552} .
$$

Substituting (2.5), (2.6), (2.8) and (2.9), and the corresponding functional form for $p_{P}(R)$, into (2.4) gives the expression for $\xi_{l}(T, R)$ as

$$
\begin{aligned}
\xi_{l}(T, R)= & \left(-0.153 T^{2}+8.61 T-97.7\right) \times\left(4 p_{M E} / R_{L}^{2}\right) R\left(R_{L}-R\right) \times\left(4 p_{M L} / R_{L}^{2}\right) R\left(R_{L}-R\right) \\
& \times e^{-(0.00554 T-0.06737)} \times\left(4 p_{M P} / R_{L}^{2}\right) R\left(R_{L}-R\right) \times\left(-0.00094 T^{2}+0.049 T-0.0552\right) \\
& \times \frac{1}{-\ln \left(-0.000828 T^{2}+0.0367 T+0.522\right)} .
\end{aligned}
$$

It is worth mentioning that all the temperature- and rainfall-dependent parameters of the model (2.1) are positive for temperature values in the range $T(t) \in[16.5,25]^{\circ} \mathrm{C}$ (the temperature range for KwaZulu-Natal province of South Africa, to be used in the numerical simulation of the model). Hence, for temperatures in this range, the temperature- and rainfall-dependent parameters of the model (2.1) are well-defined.

The non-autonomous age-structured model (2.1) is an extension of the autonomous agestructured malaria model in [23] by, inter alia:

(i) including the role of temperature and rainfall on the disease dynamics;

(ii) including separate compartments for susceptible children $\left(S_{c r}\right)$ and adults $\left(S_{a r}\right)$ with prior infection-acquired immunity;

(iii) including the dynamics of immature mosquitoes $\left(M_{a}\right)$.

Furthermore, unlike in the model developed in [23], the non-autonomous model (2.1) does not assume that infected children can carry their malaria infection to the corresponding adult class.

The model (2.1) extends the non-autonomous malaria transmission model in [1] by including the effect of rainfall (temperature was the only climatic driver considered in [1]), host age-structure and the effect of reduced susceptibility to malaria due to prior infectionacquired immunity. The model (2.1) also extends the non-autonomous malaria transmission model in [52] by including the compartment of immature mosquitoes, host age-structure and the effect of reduced susceptibility due to prior infection-acquired immunity. Unlike in [52], detailed uncertainty and sensitivity analysis of the model formulated in this study will be carried out.

It is also worth stating that the model (2.1) accounts for the conservation law of mosquito bites (so that the infection rates, $\lambda_{c}(T), \lambda_{a}(T)$ and $\lambda_{m}(T)$, are normalized by the total host population, $N_{h}(t)$; see also $\left.[8,23,24,48]\right)$.

\section{$2.2 \quad$ Basic Properties}

First of all, for simplicity, since $R=R(t)$ and $T=T(t)$, the temperature- and rainfalldependent parameters of the model will, from now on, be expressed as functions of $t$ only. 
The basic properties of the non-autonomous model (2.1) will now be explored. Let $\mu_{v}(t)=$ $\min \left\{\mu_{l}(t), \mu_{m}(t)\right\}$. Consider the equation for the rate of change of the total human $N_{h}^{\prime}(t)$ and mosquito $N_{v}^{\prime}(t)$ populations (where a prime represents differentiation with respect to time)

$$
\begin{aligned}
N_{h}^{\prime}(t) & =\Pi_{c}-\mu_{h} N_{h}-\delta_{c} I_{c}-\delta_{c r} I_{c r}-\delta_{a} I_{a}-\delta_{a r} I_{a r} \leq \Pi_{c}-\mu_{h} N_{h}, \\
N_{v}^{\prime}(t) & =\alpha_{l}(t)\left[1-\frac{M_{a}(t)}{K_{v}}\right] N_{m}(t)-\mu_{v}(t) N_{v}(t) .
\end{aligned}
$$

In the study of malaria transmission dynamics in a periodic environment (such as the model (2.1)), it is often assumed that the vector (mosquito) population stabilizes at a periodic steady-state [36]. Furthermore, following [36] it is assumed that there exists a positive number, $h_{0}$, such that

$$
\alpha_{l}(t)\left[1-\frac{M_{a}(t)}{K_{v}}\right] N_{m}(t)-\mu_{v}(t) L<0 \text { for all } L \geq h_{0} .
$$

Lemma 2.1. Consider the model (2.1) with non-negative initial conditions satisfying $N_{h}(t)>$ 0 for all $t \geq 0$. Then, the model has a unique non-negative solution in $C\left([0], \mathbb{R}_{+}^{19}\right)$ and all solutions are ultimately- and uniformly-bounded.

Proof. Following [17], the model (2.1) can be re-written as

$$
\frac{d Y}{d t}=\mathcal{A}(Y) Y+G
$$

where $Y=\left(S_{c}, S_{c r}, E_{c}, E_{c r}, I_{c}, I_{c r}, W_{c}, W_{c r}, S_{a}, S_{a r}, E_{a}, E_{a r}, I_{a}, I_{a r}, W_{a}, W_{a r}, M_{a}, M_{s}, M_{i}\right)^{T} \mathcal{A}(Y)$ is a $19 \times 19$ M-matrix (not given here to save space) and $G=\left(\Pi_{c}, 0,0,0,0,0,0,0,0,0,0,0,0\right.$, $0,0,0,0,0,0)^{T}$. It follows, using the fact that $G \geq 0$ and the matrix $\mathcal{A}(Y)$ is quasi-positive, that the system $(2.13)$ is positively-invariant in $C\left([0], \mathbb{R}_{+}^{19}\right)$. Furthermore, by comparison principle [34], that the solutions of (2.10) and (2.11) exist for all $t \geq 0$. Moreover,

$$
\limsup _{t \rightarrow \infty} N_{h}(t) \leq \frac{\Pi_{c}}{\mu_{h}}
$$

and,

$$
\limsup _{t \rightarrow \infty}\left(M_{a}(t)+M_{s}(t)+M_{i}(t)-N_{v}^{*}(t)\right) \leq 0,
$$

where $N_{v}^{*}(t)$ is the unique $\tau$-periodic solution of $(2.11)$ in $C\left([0], \mathbb{R}_{+}\right) \backslash\{0\}$, given by

$$
\begin{aligned}
N_{v}^{*}(t)= & e^{-\int_{0}^{t} \mu_{v}(s) d s} \times\left\{\int_{0}^{t}\left[\alpha_{l}(s)\left(1-\frac{M_{a}(s)}{K_{v}}\right) N_{m}(s) e^{\int_{0}^{s} \mu_{v}(\zeta) d \zeta}\right] d s\right. \\
& \left.+\frac{\int_{0}^{\tau} \alpha_{l}(s)\left(1-\frac{M_{a}(s)}{K_{v}}\right) N_{m}(s) \exp \left[\int_{0}^{w} \mu_{v}(s) d s\right]}{\exp \left[\int_{0}^{\tau} \mu_{v}(s) d s\right]-1}\right\} .
\end{aligned}
$$

Hence, all solutions of the model (2.1) are ultimately-bounded [36]. Moreover, it follows from (2.10) and (2.12) that $\frac{d N_{h}(t)}{d t}<0$ and $\frac{d N_{v}(t)}{d t}<0$ whenever $N_{h}(t)>\frac{\Pi_{c}}{\mu_{h}}$ and $N_{v}(t)>h_{0}$, respectively. Thus, all solutions of the model (2.1) are uniformly-bounded [36]. 


\section{Analysis of Autonomous Model}

It is instructive to, first of all, analyze the dynamics of the autonomous version of the nonautonomous model (2.1). Consider the special case of the non-autonomous model (2.1) where all the temperature- and rainfall-dependent parameters are constant (i.e., $\alpha_{l}(t)=\alpha_{l}, \xi_{l}(t)=$ $\left.\xi_{l}, \mu_{l}(t)=\mu_{l}, \mu_{m}(t)=\mu_{m}\right)$. The reduced model is denoted as "the autonomous mode". It is convenient to define the threshold quantity $\mathcal{N}=\frac{\alpha_{l} \xi_{l}}{\mu_{m}\left(\xi_{l}+\mu_{l}\right)}$.

The autonomous version of the model (2.1) has two disease-free equilibria, namely the trivial disease-free equilibrium (denoted by TDFE) and a non-trivial disease-free equilibrium (denoted by NDFE) as described below.

(i) If $\mathcal{N} \leq 1$, the autonomous version of the model (2.1) has a TDFE (where no mosquitoes exist) given by:

$$
\begin{aligned}
\mathcal{T}_{0} & =\left(S_{c}^{*}, S_{c r}^{*}, E_{c}^{*}, E_{c r}^{*}, I_{c}^{*}, I_{c r}^{*}, W_{c}^{*}, W_{c r}^{*}, S_{a}^{*}, S_{a r}^{*}, E_{a}^{*}, E_{a r}^{*}, I_{a}^{*}, I_{a r}^{*}, W_{a}^{*}, W_{a r}^{*}, M_{a}^{*}, M_{s}^{*}, M_{i}^{*}\right) \\
& =\left(\frac{\Pi_{c}}{\mu_{h}+\xi_{h}}, 0,0,0,0,0,0,0, \frac{\xi_{h} \Pi_{c}}{\mu_{h}\left(\mu_{h}+\xi_{h}\right)}, 0,0,0,0,0,0,0,0,0,0\right) .
\end{aligned}
$$

(ii) If $\mathcal{N}>1$, the autonomous version of the model (2.1) has a disease-free (mosquito present) equilibrium (NDFE) given by:

$$
\begin{aligned}
\mathcal{E}_{0} & =\left(S_{c}^{\diamond}, S_{c r}^{\diamond}, E_{c}^{\diamond}, E_{c r}^{\diamond}, I_{c}^{\diamond}, I_{c r}^{\diamond}, W_{c}^{\diamond}, W_{c r}^{\diamond}, S_{a}^{\diamond}, S_{a r}^{\diamond}, E_{a}^{\diamond}, E_{a r}^{\diamond}, I_{a}^{\diamond}, I_{a r}^{\diamond}, W_{a}^{\diamond}, W_{a r}^{\diamond}, M_{a}^{\diamond}, M_{s}^{\diamond}, M_{i}^{\diamond}\right) \\
& =\left(\frac{\Pi_{c}}{\mu_{h}+\xi_{h}}, 0,0,0,0,0,0,0, \frac{\xi_{h} \Pi_{c}}{\mu_{h}\left(\mu_{h}+\xi_{h}\right)}, 0,0,0,0,0,0,0, M_{a 0}, M_{s 0}, 0\right),
\end{aligned}
$$

where $M_{a 0}=\left(1-\frac{1}{\mathcal{N}}\right) K_{v}$ and $M_{s 0}=\frac{\xi_{l}}{\mu_{m}}\left(1-\frac{1}{\mathcal{N}}\right) K_{v}$.

\subsection{Asymptotic Stability of Disease-free Equilibria}

\subsubsection{TDFE}

Theorem 3.1. The TDFE of the autonomous version of the model (2.1), denoted by $\mathcal{T}_{0}$, is $G A S$ in $C\left([0], \mathbb{R}_{+}^{19}\right)$ whenever $\mathcal{N} \leq 1$.

Proof. The proof is based on using the approach in [17]. Let $\mathcal{N} \leq 1$ (so that the autonomous version of the model $(2.1)$ has only the TDFE, $\left.\mathcal{T}_{0}\right)$. Furthermore, let $Z=Y-T D F E$. Thus, Equation (2.13) can be re-written as

$$
\frac{d Z}{d t}=\mathcal{B}(Z) Z
$$

where $\mathcal{B}(Z)$ is the $19 \times 19$ matrix of coefficients of the autonomous version of the model (2.1) with variables $Z_{i}(i=1, \ldots, 19)$. It is clear that $T D F E_{Z}=(0,0,0,0,0,0,0,0,0,0,0,0,0,0,0$, $0,0,0,0)$ is the only equilibrium of the system (3.1). Consider the Lyapunov function $V(Z)=\langle W, Z\rangle$ with $W=\left(1,1,1,1,1,1,1,1,1,1,1,1,1,1,1,1, \frac{1}{\alpha_{l}}, \frac{1}{\mu_{m}}, \frac{1}{\mu_{m}}\right) \gg 0[17]$. 
Thus, $V(Z)>0$, except at $Z=T D F E_{Z}$. Furthermore (where a prime now denotes differentiation with respect to $Z$ ),

$$
\begin{aligned}
V^{\prime}(Z)= & \langle W, \mathcal{B}(Z) Z\rangle, \\
= & \left(1-\frac{Z_{17}}{K}\right)\left(Z_{18}+Z_{19}\right)-\mu_{c}\left(\sum_{i=1}^{8} Z_{i}\right)-\mu_{a}\left(\sum_{i=9}^{16} Z_{i}\right)-\frac{\xi_{l}+\mu_{l}}{\alpha_{l}} Z_{17} \\
& +\frac{\xi_{l}}{\mu_{m}} Z_{17}-Z_{18}-Z_{19}, \\
= & -\frac{Z_{17}}{K}\left(Z_{18}+Z_{19}\right)-\mu_{c}\left(\sum_{i=1}^{8} Z_{i}\right)-\mu_{a}\left(\sum_{i=9}^{16} Z_{i}\right)-(1-\mathcal{N}) \frac{\xi_{l}+\mu_{l}}{\alpha_{l}} Z_{17} .
\end{aligned}
$$

Since $\mathcal{N} \leq 1$ in $C\left([0], \mathbb{R}_{+}^{19}\right)$, it follows that $V^{\prime}(Z) \leq 0$. Furthermore, it follows from LaSalle's Invariance Principle (Theorem 6.4 of [33]) that the maximal invariant set contained in $\left\{V \mid V^{\prime}(Z)=0\right\}$ is the $T D F E_{Z}$. Thus, the transformed equilibrium, $T D F E_{Z}$, is GAS in $C\left([0], \mathbb{R}_{+}^{19}\right)$ if $\mathcal{N} \leq 1$. Hence, $\mathcal{T}_{0}$ is also GAS in $C\left([0], \mathbb{R}_{+}^{19}\right)$ whenever $\mathcal{N} \leq 1$.

It should be stated that the mosquito-free equilibrium, $\mathcal{T}_{0}$, is ecologically unrealistic (since mosquitoes always exist).

\subsubsection{NDFE}

Let $\mathcal{N}>1$ (so that the NDFE, $\mathcal{E}_{0}$, exists). The local stability of $\mathcal{E}_{0}$ in $C\left([0], \mathbb{R}_{+}^{19}\right) \backslash\left\{\mathcal{T}_{0}\right\}$, can be explored using the next generation operator method [16, 71]. Using the notation in [71], it follows that the matrices $\mathcal{F}$ (of new infection terms) and $\mathcal{V}$ (of remaining transition terms), associated with the autonomous version of the model (2.1), are given, respectively, by

$$
\mathcal{F}=\left[\begin{array}{ccccccccc}
0 & 0 & 0 & 0 & 0 & 0 & 0 & 0 & \frac{\mu_{h} b_{m} \beta_{c}}{\mu_{h}+\xi_{h}} \\
0 & 0 & 0 & 0 & 0 & 0 & 0 & 0 & 0 \\
0 & 0 & 0 & 0 & 0 & 0 & 0 & 0 & 0 \\
0 & 0 & 0 & 0 & 0 & 0 & 0 & 0 & 0 \\
0 & 0 & 0 & 0 & 0 & 0 & 0 & 0 & \frac{b_{m} \beta_{a} \xi_{h}}{\mu_{h}+\xi_{h}} \\
0 & 0 & 0 & 0 & 0 & 0 & 0 & 0 & 0 \\
0 & 0 & 0 & 0 & 0 & 0 & 0 & 0 & 0 \\
0 & 0 & 0 & 0 & 0 & 0 & 0 & 0 & 0 \\
0 & 0 & \frac{b_{m} \beta_{m} M_{s 0}}{N_{h}^{*}} & \frac{b_{m} \beta_{m} \eta M_{s 0}}{N_{h}^{*}} & 0 & 0 & \frac{b_{m} \beta_{m} M_{s 0}}{N_{h}^{*}} & \frac{b_{m} \beta_{m} \eta M_{s 0}}{N_{h}^{*}} & 0
\end{array}\right],
$$


and,

$$
\mathcal{V}=\left[\begin{array}{ccccccccc}
g_{1} & 0 & 0 & 0 & 0 & 0 & 0 & 0 & 0 \\
0 & g_{2} & 0 & 0 & 0 & 0 & 0 & 0 & 0 \\
-\sigma_{c} & 0 & g_{3} & 0 & 0 & 0 & 0 & 0 & 0 \\
0 & -\sigma_{c r} & 0 & g_{4} & 0 & 0 & 0 & 0 & 0 \\
0 & 0 & 0 & 0 & g_{5} & 0 & 0 & 0 & 0 \\
0 & 0 & 0 & 0 & 0 & g_{6} & 0 & 0 & 0 \\
0 & 0 & 0 & 0 & -\sigma_{a} & 0 & g_{7} & 0 & 0 \\
0 & 0 & 0 & 0 & 0 & -\sigma_{a r} & 0 & g_{8} & 0 \\
0 & 0 & 0 & 0 & 0 & 0 & 0 & 0 & \mu_{m}
\end{array}\right]
$$

where $N_{h}^{*}=\Pi_{c} / \mu_{h}, g_{1}=\sigma_{c}+\mu_{h}, g_{2}=\sigma_{c r}+\mu_{h}, g_{3}=\gamma_{c}+\mu_{h}+\delta_{c}, g_{4}=\gamma_{c r}+\mu_{h}+\delta_{c r}$, $g_{5}=\sigma_{a}+\mu_{h}, g_{6}=\sigma_{a r}+\mu_{h}, g_{7}=\gamma_{a}+\mu_{h}+\delta_{a}$ and $g_{8}=\gamma_{a r}+\mu_{h}+\delta_{a r}$ and . It follows from [71] that the associated basic reproduction number of the autonomous version of the model (2.1), denoted by $\mathcal{R}_{0}=\rho\left(\mathcal{F} \mathcal{V}^{-1}\right)$, is given by (where $\rho$ is the spectral radius of the next generation matrix, $\left.\mathcal{F} \mathcal{V}^{-1}\right)$

$$
\mathcal{R}_{0}=\sqrt{\frac{\mu_{h} b_{m}^{2} \beta_{m}\left(\sigma_{c} \beta_{c} g_{5} g_{7} \mu_{h}+\sigma_{a} \beta_{a} \xi_{h} g_{1} g_{3}\right)}{\Pi_{c} g_{1} g_{3} g_{5} g_{7} \mu_{m}\left(\mu_{h}+\xi_{h}\right)}\left(\frac{\xi_{l}}{\mu_{m}}\right)\left(1-\frac{1}{\mathcal{N}}\right) K_{v}}
$$

It should be noted from (3.2) that $\mathcal{R}_{0}$ is positive if and only if $\mathcal{N}>1$ (a condition for the existence of NDFE, $\mathcal{E}_{0}$ ). The result below follows from Theorem 2 of [71].

Theorem 3.2. The NDFE, $\mathcal{E}_{0}$, of the autonomous version of the model (2.1) is locallyasymptotically stable (LAS) in $C\left([0], \mathbb{R}_{+}^{19}\right) \backslash\left\{\mathcal{T}_{0}\right\}$ if $\mathcal{R}_{0}<1$, and unstable if $\mathcal{R}_{0}>1$.

\subsection{Existence of Endemic Equilibrium: Special Case}

In this section, the existence of endemic equilibrium of the autonomous version of the model (2.1) will be explored for the special case where infected recovered individual do not transmit infection (i.e. $\eta=0$ ), no disease-induced mortality in humans (i.e., $\delta_{c}=\delta_{a}=\delta_{c r}=\delta_{a r}=0$ ), so that $N_{h}^{*}=\frac{\Pi_{c}}{\mu_{h}}$. It can be shown, by solving for the variables of the resulting reduced version of the autonomous model at steady-state (and simplifying), that the solutions of the resulting model (at steady-state) satisfy the following quadratic (in terms of $\lambda_{c}^{*}$ ):

$$
c_{0}\left(\lambda_{c}^{*}\right)^{2}+c_{1}\left(\lambda_{c}^{*}\right)+c_{2}=0,
$$

where,

$$
\begin{aligned}
& c_{0}=\beta_{a} \Pi_{c} \mu_{m}^{2} g_{1} g_{3} g_{5} g_{7}, \\
& c_{1}=\Pi_{c} \mu_{m} g_{1} g_{3} g_{5} g_{7}\left\{\beta_{c} \mu_{h}+\left(\mu_{h}+\xi_{h}\right)\left[1-\left(\mathcal{R}_{0 *}\right)^{2}\right]\right\}+\beta_{a}^{2} \sigma_{a} \xi_{h} g_{1} g_{3} \mu_{h} b_{m}^{2} \beta_{m} M_{s 0}, \\
& c_{2}=\beta_{c} \mu_{h}\left[1-\left(\mathcal{R}_{0 *}\right)^{2}\right],
\end{aligned}
$$

with,

$$
\mathcal{R}_{0 *}=\left.\mathcal{R}_{0}\right|_{\delta_{c}=\delta_{a}=\delta_{c r}=\delta_{a r}=\eta=0}=\sqrt{\frac{\mu_{h} b_{m}^{2} \beta_{m}\left(\sigma_{c} \beta_{c} g_{5} g_{7} \mu_{h}+\sigma_{a} \beta_{a} \xi_{h} g_{1} g_{3}\right)}{\prod_{c} g_{1} g_{3} g_{5} g_{7} \mu_{m}\left(\mu_{h}+\xi_{h}\right)}\left(\frac{\xi_{l}}{\mu_{m}}\right)\left(1-\frac{1}{\mathcal{N}}\right) K_{v}} .
$$


Theorem 3.3. The autonomous version of the model (2.1), with $\eta=\delta_{c}=\delta_{a}=\delta_{c r}=\delta_{a r}=0$, has:

(i) a unique endemic equilibrium if $c_{2}<0 \Leftrightarrow \mathcal{R}_{0 *}>1$;

(ii) a unique endemic equilibrium if $c_{1}<0$, and $c_{2}=0$ or $c_{1}^{2}-4 c_{0} c_{2}=0$;

(iii) two endemic equilibria if $c_{2}>0, c_{1}<0$ and $c_{1}^{2}-4 c_{0} c_{2}>0$;

(iv) no endemic equilibrium otherwise.

It should be mentioned that Item (iii) of Theorem 3.3 is not feasible when $\mathcal{R}_{0 *}<1$ (since the coefficient, $c_{1}$, is positive whenever $\mathcal{R}_{0 *}<1$, so that the quadratic equation (3.3) has no positive roots in this case). Hence, for the special case in Theorem 3.3, the autonomous version of the model (2.1) does not undergo the phenomenon of backward bifurcation at $\mathcal{R}_{0 *}=1$ (see $[11,21,23,24,48,71]$ for general discussion on backward bifurcation). In fact, the following global asymptotic stability result is established for the NDFE (to completely rule out backward bifurcation in this case).

Theorem 3.4. The NDFE of the special case of the autonomous version of the model (2.1) with $\eta=\delta_{c}=\delta_{a}=\delta_{c r}=\delta_{a r}=0$ is GAS in $C\left([0], \mathbb{R}_{+}^{19}\right) \backslash\left\{\mathcal{T}_{0}\right\}$ whenever $\mathcal{R}_{0 *}<1$.

The proof of Theorem 3.4, based on using comparison theorem, is given in Appendix A. The epidemiological implication of Theorem 3.4 is that, for the special case of the autonomous version of the model (2.1) considered in Theorem 3.4, bringing (and maintaining) the threshold quantity $\mathcal{R}_{0 *}$ to a value less than unity is necessary and sufficient for the effective control (or elimination) of malaria in the population. It is worth mentioning that, as in prior models for spread of malaria and other VBDs (such as those in [11, 23, 24, 48]), the autonomous version of the model (2.1) undergoes the phenomenon of backward bifurcation if the assumption on disease-induced mortality in humans is relaxed (i.e., $\delta_{c} \neq 0, \delta_{a} \neq 0, \delta_{c r} \neq 0, \delta_{a r} \neq 0$ ). The proof of this result is not given here (to save space).

Furthermore, consider the special case of the autonomous version of model (2.1) with $\eta=\delta_{c}=\delta_{a}=\delta_{c r}=\delta_{a r}=0$ and $\mathcal{R}_{0 *}>1$ (so that the unique endemic equilibrium, denoted by $\mathcal{E}_{1}$, exists by Theorem $3.3(i)$ ). The global asymptotic stability of $\mathcal{E}_{1}$ will now be explored, for this special case. It is convenient to define:

$$
\begin{aligned}
\Omega= & \left\{\left(S_{c}, S_{c r}, E_{c}, E_{c r}, I_{c}, I_{c r}, W_{c}, W_{c r}, S_{a}, S_{a r}, E_{a}, E_{a r}, I_{a}, I_{a r}, W_{a}, W_{a r}, M_{a}, M_{s}, M_{i}\right)(t) \in C([0],\right. \\
& \left.\mathbb{R}_{+}^{19}\right) \backslash\left\{\mathcal{T}_{0}\right\}: 0<S_{c}(t) \leq S_{c}^{* *}, S_{c r}(t) \leq S_{c r}^{* *}, E_{c}(t) \leq E_{c}^{* *}, E_{c r}(t) \leq E_{c r}^{* *}, I_{c}(t) \leq I_{c}^{* *}, I_{c r}(t) \\
& \leq I_{c r}^{* *}, W_{c}(t) \leq W_{c}^{* *}, W_{c r}(t) \leq W_{c r}^{* *}, 0<S_{a}(t) \leq S_{a}^{* *}, S_{a r}(t) \leq S_{a r}^{* *}, E_{a}(t) \leq E_{a}^{* *}, E_{a r}(t) \leq \\
& E_{a r}^{* *}, I_{a}(t) \leq I_{a}^{* *}, I_{a r}(t) \leq I_{a r}^{* *}, W_{a}(t) \leq W_{a}^{* *}, W_{a r}(t) \leq W_{a r}^{* *}, 0<M_{a}(t) \leq M_{a}^{* *}, 0<M_{s}(t) \\
& \left.\leq M_{s}^{* *}, M_{i}(t) \leq M_{i}^{* *}\right\} .
\end{aligned}
$$

Since $\Omega \subset C\left([0], \mathbb{R}_{+}^{19}\right) \backslash\left\{\mathcal{T}_{0}\right\}$, it follows that $\Omega$ is positively-invariant with respcet to the aforementioned special case of the autonomous version of the model (2.1). We claim the following result.

Theorem 3.5. The unique endemic equilibrium $\left(\mathcal{E}_{1}\right)$ of the special case of the autonomous version of model (2.1) with $\eta=\delta_{c}=\delta_{a}=\delta_{c r}=\delta_{a r}=0$ is GAS in $\Omega$ whenever $\mathcal{R}_{0 *}>1$.

Proof. The proof of Theorem 3.5, based on using a non-linear Lyapunov function of GohVoltera type, is given in Appendix B. 


\section{Analysis of Non-Autonomous Model}

The non-autonomous model (2.1) has two disease-free solutions, namely the trivial diseasefree equilibrium and a non-trivial disease-free periodic solution. In this section, only the non-trivial disease-free periodic solution will be analyzed (since the former, associated with the absence of mosquitoes in the population, is ecologically unrealistic). It is convenient to define the functional threshold quantity $\mathcal{N}_{n}(t)=\frac{\alpha_{l}(t) \xi_{l}(t)}{\mu_{m}(t)\left[\xi_{l}(t)+\mu_{l}(t)\right]}$.

This (non-trivial) disease-free solution, obtained by setting $E_{c}=E_{c r}=I_{c}=I_{c r}=W_{c}=$ $W_{c r}=E_{a}=E_{a r}=I_{a}=I_{a r}=W_{a}=W_{a r}=M_{i}=0$ in (2.1), has the form (noting that $T=T(t)$ and $R=R(t))$

$$
\mathcal{E}_{0 N}=\left(S_{c}^{*}, S_{c r}^{*}, 0,0,0,0,0,0, S_{a}^{*}, S_{a r}^{*}, 0,0,0,0,0,0, M_{a}^{*}(t), M_{s}^{*}(t), 0\right)
$$

where,

$$
\left(S_{c}^{*}, S_{c r}^{*}, S_{a}^{*}, S_{a r}^{*}\right)=\left(\frac{\Pi_{c}}{\mu_{h}+\xi_{h}}, 0, \frac{\xi_{h} \Pi_{c}}{\mu_{h}\left(\mu_{h}+\xi_{h}\right)}, 0\right),
$$

with $\left(M_{a}^{*}(t), M_{s}^{*}(t)\right)$ being the unique periodic solution (for $\mathcal{N}_{n}(t)>1$ for all $t \geq 0$ ) satisfying:

$$
\begin{aligned}
\frac{d M_{a}^{*}(t)}{d t} & =\alpha_{l}(t)\left[1-\frac{M_{a}^{*}(t)}{K_{v}}\right] M_{s}^{*}(t)-\xi_{l}(t) M_{a}^{*}(t)-\mu_{l}(t) M_{a}^{*}(t), \\
\frac{d M_{s}^{*}(t)}{d t} & =\xi_{l}(t) M_{a}^{*}(t)-\mu_{m}(t) M_{s}^{*}(t) .
\end{aligned}
$$

The local asymptotic stability of $\mathcal{E}_{0 N}$ will be explored below.

\subsection{Computation of Reproduction Ratio}

The basic reproduction ratio, associated with the non-autonomous model (2.1), will be computed using the approach in [72]. The next generation matrix $F(t)$ (of the new infection terms) and the $M$-Matrix $V(t)$ (of the remaining transfer terms), associated with the nonautonomous model (2.1), are given, respectively, by

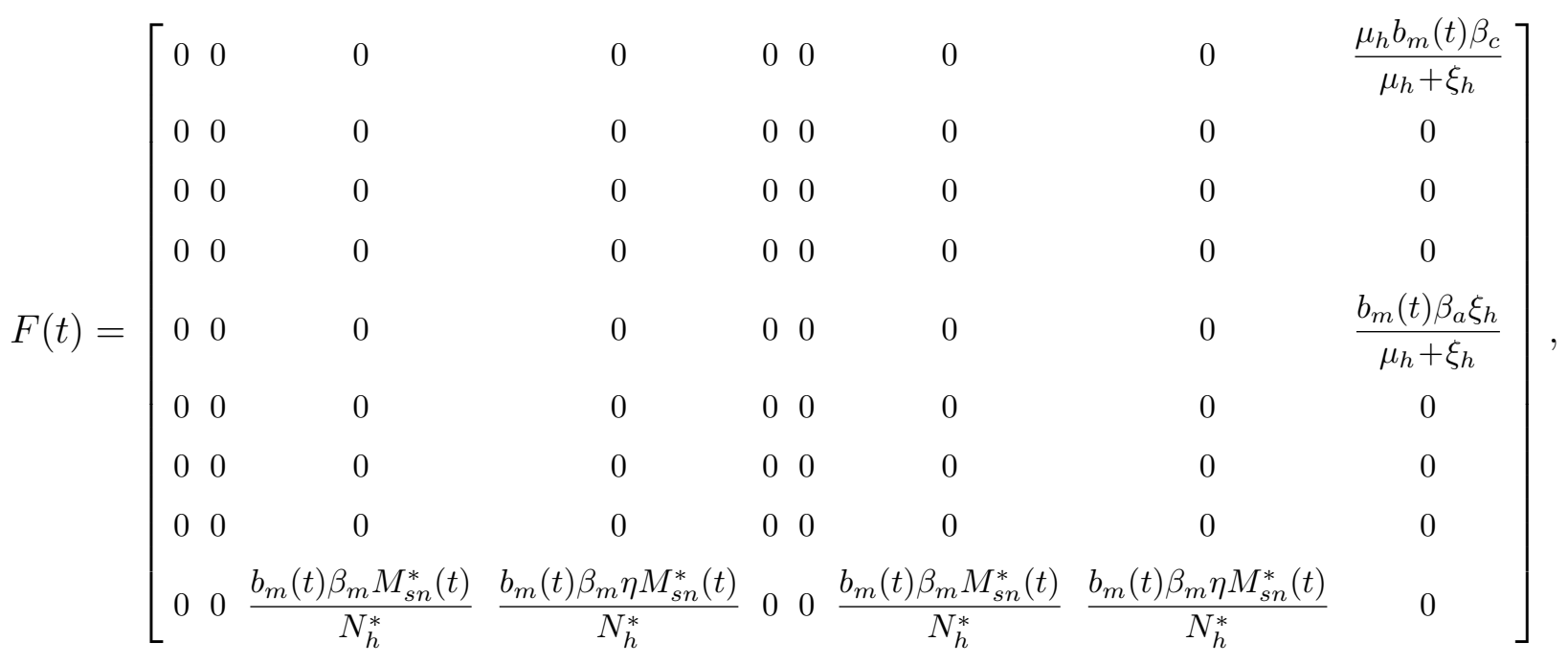


and,

$$
V(t)=\left[\begin{array}{ccccccccc}
v_{1} & 0 & 0 & 0 & 0 & 0 & 0 & 0 & 0 \\
0 & v_{2} & 0 & 0 & 0 & 0 & 0 & 0 & 0 \\
-\sigma_{c} & 0 & v_{3} & 0 & 0 & 0 & 0 & 0 & 0 \\
0 & -\sigma_{c r} & 0 & v_{4} & 0 & 0 & 0 & 0 & 0 \\
0 & 0 & 0 & 0 & v_{5} & 0 & 0 & 0 & 0 \\
0 & 0 & 0 & 0 & 0 & v_{6} & 0 & 0 & 0 \\
0 & 0 & 0 & 0 & -\sigma_{a} & 0 & v_{7} & 0 & 0 \\
0 & 0 & 0 & 0 & 0 & -\sigma_{a r} & 0 & v_{8} & 0 \\
0 & 0 & 0 & 0 & 0 & 0 & 0 & 0 & \mu_{m}(t)
\end{array}\right],
$$

where $N_{h}^{*}=\Pi_{c} / \mu_{h}, v_{1}=\sigma_{c}+\mu_{h}, v_{2}=\sigma_{c r}+\mu_{h}, v_{3}=\gamma_{c}+\mu_{h}+\delta_{c}, v_{4}=\gamma_{c r}+\mu_{h}+\delta_{c r}$, $v_{5}=\sigma_{a}+\mu_{h}, v_{6}=\sigma_{a r}+\mu_{h}, v_{7}=\gamma_{a}+\mu_{h}+\delta_{a}$ and $v_{8}=\gamma_{a r}+\mu_{h}+\delta_{a r}$. Following [72], let $\Phi_{M}$ be the monodromy matirx of the linear $\tau$-periodic system

$$
\frac{d Z}{d t}=M(t) Z
$$

Furthermore, let $\rho\left(\Phi_{M}(\tau)\right)$ be the spectral radius of $\Phi_{M}(\tau)$ and $Y(s, t), t \geq s$, be the evolution operator of the linear $\tau$-periodic system $\frac{d y}{d t}=-V(t) y$. Thus, for each $s \in \mathbb{R}$, the associated $9 \times 9$ matrix $Y(t, s)$ satisfies [72]

$$
\frac{d Y(t, s)}{d t}=-V(t) Y(t, s) \forall t \geq s, Y(s, s)=I .
$$

Suppose that $\phi(s)(\tau$-periodic in $s)$ is the initial distribution of infectious individuals. Thus, $F(s) \phi(s)$ is the rate at which new infections are produced by infected individuals who were introduced into the population at time $s$ [72]. Since $t \geq s$, it follows that $Y(t, s) F(s) \phi(s)$ represents the distribution of those infected individuals who were newly-infected at time $s$, and remain infected at time $t$. Hence, the cumulative distribution of new infections at time $t$, produced by all infected individuals $(\phi(s))$ introduced at a prior time $s=t$, is given by

$$
\Psi(t)=\int_{-\infty}^{t} Y(t, s) F(s) \phi(s) d s=\int_{0}^{\infty} Y(t, t-a) F(t-a) \phi(t-a) d a .
$$

Let $\mathbb{C}_{\tau}$ be the ordered Banach space of all $\tau$-periodic functions from $\mathbb{R}$ to $\mathbb{R}^{9}$, which is equipped with maximum norm and positive cone $\mathbb{C}_{\tau}^{+}\left\{\phi \in \mathbb{C}_{\tau}: \phi(t) \geq 0, \forall t \in \mathbb{R}\right\}$ [72]. Define a linear operator $L: \mathbb{C}_{\tau} \rightarrow \mathbb{C}_{\tau}[72]$

$$
(L \phi)(t)=\int_{0}^{\infty} Y(t, t-a) F(t-a) \phi(t-a) d a \forall t \in \mathbb{R}, \phi \in \mathbb{C}_{\tau} .
$$

The basic reproduction ratio $\left(\mathcal{R}_{0 T R}\right)$ is then given by the spectral radius of $L$, denoted by $\rho(L)$. That is, $\mathcal{R}_{0 T R}=\rho(L)$. It can be verified that system (2.1) satisfy the assumptions $A 1-A 7$ in [72]. Hence, the result below follows from Theorem 2.2 in [72]

Theorem 4.1. Let $\mathcal{N}_{n}(t)>1$ for all $t \geq 0$. The DFS $\left(\mathcal{E}_{0 N}\right)$, of the non-autonomous model (2.1), is LAS if $\mathcal{R}_{0 T R}<1$ and unstable if $\mathcal{R}_{0 T R}>1$. 
The global asymptotic stability of the DFS, $\mathcal{E}_{0 N}$, is now considered for a special case.

Theorem 4.2. Consider a special case of the non-autonomous model (2.1) with $\delta_{c}=\delta_{a}=$ $\delta_{c r}=\delta_{a r}=0$ (so that $N_{h}^{*}(t) \rightarrow \Pi_{c} / \mu_{h}$ as $t \rightarrow \infty$ ). Let $\mathcal{N}_{n}(t)>1$ for all $t \geq 0$. The DFS of the resulting model is $G A S$ in $C\left([0], \mathbb{R}_{+}^{19}\right) \backslash\left\{\mathcal{T}_{0}\right\}$ if $\mathcal{R}_{0 T R *}=\left.\mathcal{R}_{0 T R}\right|_{\delta_{c}=\delta_{a}=\delta_{c r}=\delta_{a r}=0}<1$.

The proof of Theorem 4.2, based on using comparison theorem [63], is given in Appendix C. The epidemiological implication of Theorem 4.2 is that malaria can be effectively controlled (or eliminated) if the reproduction threshold $\mathcal{R}_{0 T R *}$ can be brought to (and maintained at) a value less than unity.

\subsection{Uniform Persistence of the Disease}

In this section, the possibility of the existence of a positive periodic solution for the system (2.1) will be explored using uniform persistence theory [36, 39, 69, 77, 78, 79]. Let $\mathcal{E}_{N}^{*}(t)$ represent any arbitrary positive periodic solution of the model (2.1). Following [36], it is convenient to define the following sets:

$$
\begin{aligned}
X= & C\left([0], \mathbb{R}_{+}^{19}\right), \\
X_{0}= & \left\{\phi=\left(\phi_{1}, \phi_{2}, \phi_{3}, \phi_{4}, \phi_{5}, \phi_{6}, \phi_{7}, \phi_{8}, \phi_{9}, \phi_{10}, \phi_{11}, \phi_{12}, \phi_{13}, \phi_{14}, \phi_{15}, \phi_{16}, \phi_{17}, \phi_{18}, \phi_{19}\right) \in X:\right. \\
& \left.\phi_{i}(0)>0 \text { for all } i \in\{2,3,4,5,6,7,8,10,11,12,13,14,15,16,19\}\right\} .
\end{aligned}
$$

Theorem 4.3. Consider the non-autonomous model (2.1). Suppose $\mathcal{R}_{0 T R}>1$ and $\mathcal{N}_{n}(t)>$ 1 for all $t \geq 0$. Then the model has at least one positive periodic solution, and there exists a $q>0$ such that any solution $\Phi_{t}(\phi)$ of the model with initial value $\phi \in X_{0}$ satisfies

$$
\begin{aligned}
& \liminf _{t \rightarrow \infty} E_{c} \geq q, \liminf _{t \rightarrow \infty} E_{c r} \geq q, \liminf _{t \rightarrow \infty} I_{c} \geq q, \liminf _{t \rightarrow \infty} I_{c r} \geq q, \liminf _{t \rightarrow \infty} R_{c} \geq q, \liminf _{t \rightarrow \infty} R_{c r} \geq q, \\
& \liminf _{t \rightarrow \infty} E_{a} \geq q, \liminf _{t \rightarrow \infty} E_{a r} \geq q, \liminf _{t \rightarrow \infty} I_{a} \geq q, \liminf _{t \rightarrow \infty} I_{a r} \geq q, \liminf _{t \rightarrow \infty} R_{a} \geq q, \liminf _{t \rightarrow \infty} R_{a r} \geq q .
\end{aligned}
$$

The proof of Theorem 4.3, based on using the approach in [36], is given in Appendix D. The epidemiological implication of Theorem 4.3 is that malaria will persist in the population whenever $\mathcal{R}_{0 T R}>1$ and $\mathcal{N}_{n}(t)>1$ for all $t \geq 0$.

\section{$5 \quad$ Uncertainty and Sensitivity Analysis}

The non-autonomous model (2.1) has 31 parameters, and uncertainties are expected to arise in the estimates of their values used in the numerical simulations. The effect of these uncertainties, as well as the determination of the parameters that have the greatest influence on the transmission dynamics of the disease (with respect to a given response function), are carried out using an uncertainty and sensitivity analysis [1, 6, 41, 43, 44]. In particular, following [6], Latin Hypercube Sampling (LHS) and Partial Rank Correlation Coefficients (PRCC) will be used for both the autonomous equivalent and the non-autonomous model as below. The analyses will be carried out using data relevant to malaria transmission dynamics in KwaZulu-Natal province of South Africa. 


\subsection{Autonomous Model}

In this section, uncertainty and sensitivity analysis will be carried out on the autonomous version of the model (2.1), where all temperature- and rainfall-dependent parameters of the model are assumed to be constant. The range and baseline values of the parameters, tabulated in Table 2, will be used. Appropriate response functions are chosen for these analyses.

Using the population of infectious humans with no prior immunity $\left(I_{c}\right.$ and $\left.I_{a}\right)$ as the response functions, it is shown in Table 3 that the top three PRCC-ranked parameters of the autonomous version of the model are the mosquito carrying capacity $\left(K_{v}\right)$, the biting rate $\left(b_{m}\right)$ and transmission probability per contact for susceptible mosquitoes $\left(\beta_{m}\right)$. Similarly, using the population of infectious individuals with prior immunity $\left(I_{c r}\right.$ and $\left.I_{a r}\right)$ as the response functions, the top three PRCC-ranked parameters are the mosquito carrying capacity $\left(K_{v}\right)$, the biting rate $\left(b_{m}\right)$ and the corresponding recovery rates of children and adults with no prior immunity to malaria $\left(\gamma_{c}\right.$ and $\left.\gamma_{a}\right)$ respectively. Furthermore, using the population of infected mosquitoes $\left(M_{i}\right)$ as the response function, it is shown that the same top three PRCC-ranked parameters appeared as in the case when infectious humans with no prior immunity are chosen as the response functions. This result also holds when the basic reproduction number of the autonomous version of the model $\left(\mathcal{R}_{0}\right)$ is chosen as the response function. In summary, this study identifies four parameters that dominate the transmission dynamics of the autonomous version of the model, namely mosquito carrying capacity, biting rate, transmission probability per contact for susceptible mosquitoes and the recovery rates of infectious with no prior immunity to malaria infection.

\subsection{Non-autonomous Model}

Uncertainty and sensitivity analysis is also carried out for the non-autonomous model (2.1) using the data for the ranges and baseline values of the temperature- and rainfall-dependent parameters of the model tabulated in Tables 5 and 6 . Using the basic reproduction ratio $\left(\mathcal{R}_{0 T R}\right)$ as the response function, it is shown (Table 7 ) that, for all temperature and rainfall values, the top three PRCC-ranked parameters are the mosquito carrying capacity $\left(K_{v}\right)$, transmission probability per contact for susceptible mosquitoes $\left(\beta_{m}\right)$ and recovery rate of adults with no prior immunity $\left(\gamma_{a}\right)$. For the case when the total population of infectious humans with no prior immunity $\left(I_{a}+I_{c}\right)$ is chosen as the response function, the top three PRCC-ranked parameters are (Table 8$)$ the mosquito carrying capacity $\left(K_{v}\right)$, recovery rates for human with no prior immunity $\left(\gamma_{c}\right.$ and $\left.\gamma_{a}\right)$ and transmission probability per contact for susceptible mosquitoes $\left(\beta_{m}\right)$.

It is worth noting that the non-autonomous model (2.1) and its autonomous equivalent have two top PRCC-ranked parameters in common $\left(K_{v}\right.$ and $\left.\beta_{m}\right)$. While the biting rate $\left(b_{m}\right)$ is ranked highly (top three) in the autonomous case, this rate does not feature among the top three PRCC-ranked parameters of the non-autonomous model. 


\section{Numerical Simulations}

The non-autonomous model (2.1) is simulated to illustrate the effect of the two climate variables (temperature and rainfall) on the transmission dynamics of malaria in the population (KwaZulu-Natal Province of South Africa). In the numerical simulations, the ranges and baseline values of the temperature- and rainfall-dependent parameters of the model are computed using their respective expressions in Section 2.1 (and the numerical values tabulated in Tables 5 and 6). Demographic parameters relevant to KwaZulu-Natal were chosen. In particular, since the total population of KwaZulu-Natal is estimated to be 10,267,300 [64], it follows that, at disease-free steady-state, $\Pi_{c} / \mu_{h}=10,267,300$. Furthermore, since the average lifespan in South Africa is 51-53 years [70], the parameter $\mu_{h}$ is given as $\mu_{h} \in[1 / 53,1 / 51$ ] per year, so that $\Pi_{c} \in[530,552]$ per day. It is worth noting that all temperature- and rainfall dependent parameters are positive for the temperature range $T(t) \in[16.5,35.6]^{\circ} \mathrm{C}$.

The effect of mean monthly temperature on the transmission dynamics of the disease is, first of all, evaluated by simulating the model (2.1) at a fixed mean monthly rainfall value (namely the mean monthly rainfall value for KwaZulu-Natal, $R(t)=86.63 \mathrm{~mm}[18]$ ). The results obtained, depicted in Figure 3 (see also Table 5 for the values of the temperaturedependent parameters of the model), show that the total number of new malaria cases increases with increasing mean monthly temperature until a peak of $[24-25]^{\circ} \mathrm{C}$ is reached (which occurs between December to March), and the total number of cases decreases with decreasing temperature thereafter. This suggests that, for the KwaZulu-Natal data, malaria transmission is maximum at mean monthly temperatures in the range $[24-25]^{\circ} \mathrm{C}$ (this result is consistent with the finding in [1]). The mean monthly temperature data in Table 4 shows that KwaZulu-Natal experience this suitable temperature, for maximum malaria activity, for a period of four months (between December and March).

The effect of mean monthly rainfall, at a fixed mean monthly temperature $\left(T(t)=21.5^{\circ} \mathrm{C}\right.$ [18]), is also monitored. Figure 4 shows an increase in malaria cases with increasing rainfall until an initial peak rainfall value (of $R(t)=107 \mathrm{~mm}$ ) is reached in October (this corresponds to an increase in mosquito maturation rate, as shown in Table 6). The increase in rainfall between December to February (where $R(t) \in[118-142] \mathrm{mm}$ ) resulted in a corresponding decrease in malaria cases (and also a corresponding decrease in mosquito maturation rate). Further decrease in rainfall between March and April resulted in a concomitant increase in the total number of malaria cases.

It is worth stating that very low mean monthly rainfall values, such as the $[32-65] \mathrm{mm}$ range recorded between May to September (Figure 4), lead to a greatly reduced number of malaria cases (as expected). In summary, Figure 4 and Table 6 show that, under the condition of the mean monthly temperature for KwaZulu-Natal, malaria transmission is maximized if the mean monthly rainfall is in the range [95 - 125] $\mathrm{mm}$ (which occurs for six-month period, in October, November, December, January, March and April). These simulations show that malaria transmission in KwaZulu-Natal is maximized when the mean monthly temperature and rainfall values are at the ranges $[21-25]^{\circ} \mathrm{C}$ and $[95-125] \mathrm{mm}$, respectively. Figures 3 and 4 (as well as Tables 5 and 6) show that such suitable mean monthly temperature and rainfall values occur simultaneously in at least six months of the year in KwaZulu-Natal (namely, during the months of October, November, December, January, March and April). Hence, this study suggests that anti-malaria control efforts 
should be intensified during these months. This result is consistent with the finding in [14] (a statistical study which shows that, for the assumption of a mean monthly temperature, five months of rainfall above $80 \mathrm{~mm}$ is sufficient for stable malaria transmission) the KwaZuluNatal.

The combined effect of mean monthly temperature and rainfall is further assessed by simulating the model (2.1) using various combinations of temperature and rainfall values, as tabulated in Table 4. The results obtained, depicted in Figure 5, show similar trends as those observed in Figures 3 and 4 (with malaria burden increasing with increasing temperature and rainfall until a peak is reached, and decreasing with decreasing mean monthly temperature and rainfall thereafter). Furthermore, it follows from Tables 3, 7 and 8 that the parameters related to the host age-structure component of the model (2.1) (that is, the maturation rate of children to adult $\left(\xi_{h}\right)$ ) and reduced susceptibility due prior malaria infection (namely, the infection-acquired immunity rate for children $\left(\psi_{c}\right)$ and adults $\left(\psi_{a}\right)$ ) have very low PRCC ranking. Hence, this study shows that adding host age-structure and reduced susceptibility to malaria infection due to prior immunity has marginal effect on the disease transmission dynamics (see also Figures 6a and 6b).

\section{Conclusions}

A new deterministic, non-autonomous, age-structured model for assessing the impact of temperature and rainfall variability on the transmission dynamics of malaria in a population is designed and analyzed. The main theoretical and epidemiological findings of this study are summarized below:

(i) The autonomous version of the model has a trivial (mosquito-free) equilibrium which is globally-asymptotically stable whenever a certain threshold quantity $(\mathcal{N})$ is less than unity. It also has a mosquito-present disease-free equilibrium which is globallyasymptotically stable, for a special case without disease-induced mortality in humans, whenever the associated reproduction number is less than unity. For the case when the reproduction number exceeds unity, the model is shown to have a unique endemic equilibrium for a special case (with $\eta=\delta_{c}=\delta_{c r}=\delta_{a}=\delta_{a r}=0$ ). This unique equilibrium is globally-asymptotically stable in (in $\Omega$ ) when it exists.

(ii) The disease-free periodic solution of the non-autonomous model is shown to be globalyasymptoticaly stable, in the absence of disease-induced mortality for humans, whenever the spectral radius of a certain linear operator (denoted by $\mathcal{R}_{0 T R *}$ ) is less than unity. The model has at least one periodic solution whenever $\mathcal{R}_{0 T R}>1$ (and the disease persists in the population in this case).

Uncertainty and sensitivity analysis of the autonomous version and the non-autonomous model show the following:

(a) For the autonomous model, using the population of infectious humans with no prior immunity $\left(I_{c}\right.$ and $\left.I_{a}\right)$ as the response functions, it is shown in Table 3 the top three PRCCranked parameters of the autonomous version of the model (2.1) are the mosquito carrying capacity $\left(K_{v}\right)$, biting rate $\left(b_{m}\right)$ and transmission probability per contact for 
susceptible mosquitoes $\left(\beta_{m}\right)$. Similarly, using the population of infectious individuals with prior immunity $\left(I_{c r}\right.$ and $\left.I_{a r}\right)$ as the response function, the top three PRCC-ranked parameters are the mosquito carrying capacity $\left(K_{v}\right)$, mosquito biting rate $\left(b_{m}\right)$ and the corresponding recovery rates of children and adults with no prior immunity $\left(\gamma_{c}\right.$ and $\left.\gamma_{a}\right)$. Furthermore, using the population of infected mosquitoes $\left(M_{i}\right)$ as the response function, it is shown that the same top three PRCC-ranked parameters appeared as in the case when infectious humans with no prior immunity are chosen as the response function. This result also holds when the basic reproduction number of the autonomous version of the model $\left(\mathcal{R}_{0}\right)$ is chosen as the response function.

(b) For the non-autonomous model, using the basic reproduction ratio $\left(\mathcal{R}_{0 T R}\right)$ as the response function, it is shown (Table 7) that, for all temperature and rainfall values, the top three PRCC-ranked parameters are the mosquito carrying capacity $\left(K_{v}\right)$, transmission probability per contact for susceptible mosquitoes $\left(\beta_{m}\right)$ and recovery rate of adults with no prior immunity $\left(\gamma_{a}\right)$. For the case when the total population of infectious humans with no prior immunity $\left(I_{a}+I_{c}\right)$ is chosen as the response function, the top three PRCC-ranked parameters are the mosquito carrying capacity $\left(K_{v}\right)$, recovery rates of humans with no prior immunity $\left(\gamma_{c}\right.$ and $\left.\gamma_{a}\right)$ and transmission probability per contact for susceptible mosquitoes $\left(\beta_{m}\right)$ (Table 8$)$.

(c) The mosquito maturation rate $\left(\xi_{l}\right)$ and the biting rate $\left(b_{m}\right)$ have maximum $\mathrm{PRCC}$ values at mean monthly temperature and rainfall ranges given by $T(t) \in[16,20]^{\circ} \mathrm{C}$ and $R(t) \in[60-120] \mathrm{mm}$. The maximum PRCC value for the transmission probability per contact for susceptible mosquitoes $\left(\beta_{m}\right)$ occurs at $T(t) \in[21-25]^{\circ} \mathrm{C}$ and $R(t) \in[90-$ $120] \mathrm{mm}$. Hence, in these temperature and rainfall ranges, there is significant malaria activity (suggesting a heightened anti-malaria control, particularly mosquito-reduction and personal protection against mosquito bites during this period). In other words, this study shows that malaria activity is maximized in the province of KwaZulu-Natal between October and April (except February) when the climatic condition $(T(t) \in$ $[21-25]^{\circ} \mathrm{C}$, and $R(t) \in[95-125] \mathrm{mm}$ ) are most favourable for malaria activity.

Numerical simulations of the non-autonomous model, using relevant data from KwaZuluNatal Province of South Africa, show the following:

(i) For a fixed mean monthly rainfall value of KwaZulu-Natal $(R(t)=86.63 \mathrm{~mm})$, malariaassociated burden (measured in terms of the total number of new cases) increases with increasing mean monthly temperature in the recorded range for KwaZulu-Natal $\left([17-25]^{\circ} \mathrm{C}\right.$ and decreases with decreasing mean monthly temperature (this result supports the finding in [45]);

(ii) For a fixed mean monthly temperature value of KwaZulu-Natal $\left(T(t)=21.5^{\circ} \mathrm{C}\right)$, malaria-associated burden increases with increasing rainfall value until a threshold of $[95-110] \mathrm{mm}$ is reached. At higher rainfall values (above the threshold), disease burden decreases. This is as a result of the decreasing maturation rate (above the threshold) due to flushing out of immature mosquitoes from the breeding sites of mosquitoes (this result supports the finding in [50]); 
(iii) Malaria-associated burden is maximized when the mean monthly temperature values are in the $[21-25]^{\circ} \mathrm{C}$ range and the mean monthly rainfall values are in the range $[95-$ 125] mm. This occurs during six months of the year (October, November, December, January, March and April). Thus, this study suggests that anti-malaria control efforts should be intensified for KwaZulu-Natal during these months (this result supports the finding in [14]);

(iv) The total number of new cases of malaria infection increases with increasing maturation rate of mosquitoes;

(v) Incorporating age-structure and reduced susceptibility due to prior infection to models for malaria transmission dynamics have marginal effect on the disease dynamics (this result supports the finding in [23]).

\section{Acknowledgments}

One of authors (ABG) is grateful to National Institute for Mathematical and Biological Synthesis (NIMBioS) for funding the Working Group on Climate Change and Vector-borne Diseases (VBDs). NIMBioS is an Institute sponsored by the National Science Foundation, the U.S. Department of Homeland Security, and the U.S. Department of Agriculture through NSF Award \#EF-0832858, with additional support from The University of Tennessee, Knoxville. ABG acknowledges, with thanks, the support, in part, of the Natural Sciences and Engineering Research Council (NSERC) of Canada. The authors are grateful to the anonymous reviewers for their constructive comments. 


\section{References}

[1] Agusto, F.B., Gumel, A.B. and Parham, P.E. Qualitative assessment of the role of temperature variations on malaria transmission dynamics. Journal of Biological Systems. 23(4)(2015): 1 - 34 .

[2] Alonso, D., Bouma, M.J. and Pascual, M. Epidemic malaria and warmer temperatures in recent decades in an East African highland. Proceedings of the Royal Society $B$. 278(2011): 1661-1669. doi:10.1098/rspb.2010.2020.

[3] Baeza, A., Bouma, M. J., Dhiman, R. and Pascual, M. Malaria control under unstable dynamics: reactive vs. climate-based strategies. Acta Tropica129(2014): 42-51.

doi:10.1016/j.actatropica.2013.04.001.

[4] Beck-Johnson, L.M., Nelson, W.A., Paaijmans, K.P., Read, A.F., Thomas, M.B. and Bjornstad, O.N. The effect of temperature on Anopheles mosquito population dynamics and the potential for malaria transmission. PLOS ONE. 8(2013):11. e79276.

[5] Blanford, J.I., Blanford, S., Crane, R.G., Mann, M.E., Paaijmans, K.P., Schreiber, K.V., and Thomas, M.B. Implications of temperature variation for malaria parasite development across Africa. Scientific Reports. 1300(2013). doi: 10.1038/srep01300.

[6] Blower, S.M. and Dowlatabadi, H. Sensitivity and uncertainty analysis of complex models of disease transmission: an HIV model, as an example. International Statistical Review. 2(1994): 229-243.

[7] Bouma, M.J., Baeza, A., terVeen, A. and Pascual, M. Global malaria maps and climate change: a focus on East African highlands. Trends in Parasitology. 27(2011): 421-422.

[8] Bowman, C., Gumel, A.B., Van den Driessche, P., Wu, J. and Zhu, H. A mathematical model for assessing control strategies against West Nile virus. Bulletin of Mathematical Biology. 67(2005): 1107-1133.

[9] Boyd, M.F. Epidemiology of Malaria: Factors Related to the Definitive Host. In Malariology: A Comprehensive Survey of all Aspects of this Group of Diseases from a Global Standpoint (ed. MF Boyd). Philadelphia, PA: W.B. Saunders Company. (1949): 608697.

[10] Cash, B., Rodó, X., Ballester, J., Bouma, M.J., Baeza, A., Dhiman, R.C. and Pascual, M. Malaria epidemics highlight the influence of the Tropical South Atlantic on the Indian monsoons. Nature Climate Change 3(2013): 502-207. doi:10.1038/nclimate1834.

[11] Castillo-Chavez, C. C., and Song, B. Dynamical models of tuberculosis and their applications. Mathematical Biosciences and Engineering. 1(2004): 361-404.

[12] Chitnis, N., Cushing, J.M. and Hyman, J.M. Bifurcation analysis of a mathematical model for malaria transmission. SIAM Journal on Applied Mathematics. 67(2006): 2445 . 
[13] Coutinho, F.A.B., Burattini, M.N., Lopez, L.F. and Massad, E. An approximate threshold condition for non-autonomous system: An application to a vector-borne infection. Mathematics and Computers in Simulation. 70(2005): 149-158.

[14] Craig, M.H., Snow R.W. and Le Sueur, D. Scholarly articles for A climate-based distribution model of malaria transmission in Sub-Saharan Africa. Parasitology Today. 15(2005): 149-158.

[15] Dentinova T.S. Age-grouping methods in diptera of medical importance. Geneva, Switzerland: World Health Organisation. (1962).

[16] Diekmann, O., Heesterbeek, J. and Metz, J. On the definition and the computation of the basic reproduction ratio $R_{0}$ in models for infectious diseases in heterogeneous populations. Journal of Mathematical Biology. 28(1990): 365-382.

[17] Dumont, Y. and Chiroleu, F. Vector control for the Chikungunya disease. Mathematical Biosciences and Engineering. 7(2010): 105-111.

[18] Durban Monthly Climate Average, South Africa.

http://www.worldweatheronline.com/Durban-weather-averages/Kwazulu-

Natal/ZA.aspx. Accessed: June 2015.

[19] Edwin, M. and Robert, C. Modeling parasite transmission and control. Advances in Experimental Medicine and Biology. 673(2010).

[20] Egbendewe-Mondzozo, A., Musumba, M., McCarl, B.A. and Wu, X. (2011). Climate change and vector-borne diseases: an economic impact analysis of malaria in Africa. International Journal Environmental Research and Public Health. 8 (2011): 913-930.

[21] Elbasha, E.H. and Gumel, A.B. Theoretical assessment of public health impact of imperfect prophylactic HIV-1 vaccines with therapeutic benefits. Bulletin of Mathematical Biology. 7(2006): 577-614.

[22] Faye, O., Gaye, O., Fontenille, D., Hébrard, G., Konate, L., Sy, N., Herve, J.P., Toure, Y.T., Diallo, S. and Molez, J.F. Malaria decrease and drought in the Niayes area of Senegal. Sante 5(1995): 299-305.

[23] Forouzannia, F. and Gumel, A.B. Mathematical analysis of an age-structured model for malaria transmission dynamics. Mathematical Biosciences. 247(2014): 80-94.

[24] Garba, S. M., Gumel, A.B. and Abu Bakar, M.R. Backward bifurcations in dengue transmission dynamics. Mathematical Biosciences. 215(2008): 11-25.

[25] Garms, R., Walsh, J.F., Davies, J.B. Studies on the reinvasion of the onchocerciasis control programme in the Volta River Basin by Simulium damnosum s.I. with emphasis on the south-western areas. Tropenmedizin und Parasitologie. 30(1979): 345-362.

[26] Githeko, A.K., Lindsay, S.W., Confalonieri, U.E. and Patz, J.A. Climate change and vector-borne diseases: a regional analysis. Bulletin of the World Health Organization. 78(2000): 9 . 
[27] Health and Environment Linkages Initiative - HELI. World Health Organization. http://www.who.int/heli/risks/vectors/vector/en/. Accessed: June 2015.

[28] Horsfall W.R. Mosquitoes: their bionomics and relation to disease. Hafner Publishing Company, New York. 1955.

[29] Hoshen, M.B. and Morse, A.P. A weather-driven model of malaria transmission. Malaria Journal. 3(2004). 32. (doi:10.1186/1475-2875-3-32)

[30] Imbahale, S.S., Paaijmans, K.P., Mukabana, W.R., van Lammeren, R., Githeko, A.K. and Takken, W. A longitudinal study on Anopheles mosquito larval abundance in distinct geographical and environmental settings in western Kenya. Malaria Journal. 10(2011): 81 .

[31] Jepson, W.F., Moutia, A., and Courtois, C. The malaria problem in Mauritius: the bionomics of Mauritian anophelines. Bulletin of Entomology Res. 38(1947): 177-208.

[32] Lafferty K.D. The ecology of climate change and infectious diseases. Ecology. 90(2009): 888 - 900. (doi:10.1890/08-0079.1).

[33] J.P. LaSalle. The Stability of Dynamical Systems. Regional Conference Series in Applied Mathematics. SIAM Philadephia. 1976.

[34] Lakshmikantham, V. and Leela, S. Differential and Integral Inequalities: Theory and Applications. Academic Press, New York.

[35] Laperriere, V., Brugger, K. and Rubel, F. Simulation of the seasonal cycles of bird, equine and human West Nile virus cases. Preventive Veterinary Medicine. 88(2011): 99-110.

[36] Lou, Y. and Zhao, X.-Q. A climate-based malaria transmission model with structured vector population. SIAM Journal on Applied Mathematics. 70(2010): 2023-2044.

[37] Lunde, T.M., Bayoh, M.N. and Lindtjorn, B. How malaria models relate temperature to malaria transmission. Parasites $\&$ Vectors 6(2013): 20.

http://www.parasitesandvectors.com/content/6/1/20.

[38] Macdonald, G. The Epidemiology and Control of Malaria. London, UK: Oxford University Press. (1957).

[39] Magal, P.and Zhao, X.-Q. Global attractors and steady states for uniformly persistent dynamical systems. SIAM Journal on Mathematical Analysis . 37(2005): 251-275.

[40] MARA/ARMA. Towards an Atlas of Malaria Risk in Africa. Albany Print Ltd. Durban, South Africa. (1998): 1-31.

[41] Marino, S., Hogue, I.B., Ray, C.J. and Kirschner, D.E. A methodology for performing global uncertainty and sensitivity analysis in systems biology. Journal of Theoretical Biology. 254(2008): 178-196. 
[42] Martens, W.J. Health impacts of climate change and ozone depletion: an ecoepidemiologic modeling approach. Environmental Health Perspectives. 106(1998)(Suppl. 1), 241251. (doi:10.1289/ehp. 98106s1241).

[43] Mckay, M.D., Beckman, R.J., and Conover, W.J. Comparison of 3 methods for selecting values of input variables in the analysis of output from a computer code. Technometrics. 21 (1979): 239-245.

[44] McLeod, R.G., Brewster, J.F., Gumel, A.B., and Slonowsky, D.A. Sensitivity and uncertainty analyses for a SARS model with time-varying inputs and outputs. Mathematical Biosciences and Engineering. 2(2006): 527-544.

[45] Mordecai, E.A. et al. Optimal temperature for malaria transmission is dramatically lower than previously predicted. Ecology Letters. 16(2013): 22-30.

[46] Murdock, C.C., Paaijmans, K.P., Bell, A.S., King, J.G., Hillyer, J.F., Read, A.F. and Thomas, M.B. Complex effects of temperature on mosquito immune function. Proceedings. Biological Science / The Royal Society. 279(2012) 3357-3366. (doi:10.1098/rspb. 2012.0638).

[47] Murdock, C.C., Paaijmans, K.P., Cox-Foster, D., Read, A.F., and Thomas, M.B. Rethinking vector immunology: the role of environmental temperature in shaping resistance. Nature Reviews. Microbiology. 10(2012): 869-876. (doi:10.1038/nrmicro2900).

[48] Niger, A.M. and Gumel, A.B. Mathematical analysis of the role of repeated exposure on malaria transmission dynamics. Differential Equations and Dynamical Systems. 16(2008): 251-287.

[49] Paaijmans, K.P., Imbahale, S.S., Thomas, M.B. and Takken, W. Relevant microclimate for determining the development rate of malaria mosquitoes and possible implications of climate change. Malaria Journal. 9(2010): 196. (doi:10.1186/1475-2875-9-196)

[50] Paaijmans, K.P., Wandago, M.O., Githeko, A.K. and Takken, W. Unexpected high losses of Anopheles gambiae larvae due to rainfall. PLOS One. 2(2007).

[51] Parham, P.E., Michael, E. Modelling climate change and malaria transmission. Advances in Experimental Medicine and Biology. 673(2010): 184 - 199. (doi:10.1007/978-1-44196064-1_13).

[52] Parham, P.E. and Michael, E. Modeling the effects of weather and climate change on malaria transmission. Environmental Health Perspectives. 118(2010): 620-626. (doi:10.1289/ehp.0901256).

[53] Parham, P.E., Pople, D., Christiansen-Jucht, C., Lindsay, S., Hinsley, W. and Michael, E. Modeling the role of environmental variables on the population dynamics of the malaria vector Anopheles gambiae sensu stricto. Malaria Journal. 11 (2012): 271. 
[54] Parham, P.E., Waldock, J., Christophides, K.G., Hemming, D., Agusto, F., Evans, J.K., Fefferman, N., Gaff, H., Gumel, A.B., LaDeau, S., Lenhart, S., Mickens, R.E., Naumova, E., Ostfeld R., Ready, P., Thomas, M, Velasco-Hernandez, J. and Michael, E. Climate, environmental, and socioeconomic change - weighing up the balance in vector-borne disease transmission. Philosophical Transaction of the Royal Society B. 370(2015): 20130551. http://dx.doi.org/10.1098/rstb.2013.0551.

[55] Pilgrim, J.M. Fang, X. and Stefan, H.G. Correlations of Minnesota Stream Water Temperatures with Air Temperatures. Project Report 382, prepared for National Agricultural Water Quality Laboratory Agricultural Research Service U. S. Department of Agriculture Durant, Oklahoma (1995).

[56] Preud'homme, E.B. and Stefan, H.G. Relationship BetweenWater Temperatures and Air Temperatures for Central U. S. Streams. Project Report No. 333, prepared for Environmental Research Laboratory U.S. Environmental Protection Agency Duluth, Minnesota (1992).

[57] Rodó, X. et al. Climate change and infectious diseases: can we meet the needs for better prediction? Climate Change. 118(2013): 625 - 640. (doi:10.1007/s10584-013-0744-1)

[58] Rogers, D.J. and Randolph, S.E. The global spread of malaria in a future, warmer world. Science. 289(2000): 1763-1767.

[59] Rohr, J.R., Dobson, A.P., Johnson, P. T., Kilpatrick, A.M., Paull, S.H., Raffel, T.R., Ruiz-Moreno, D. and Thomas, M.B. Frontiers in climate change disease research. Trends in Ecology and Evolution. 26(2011): 270-277.

[60] Rubel, F., Brugger, K., Hantel, M., Chvala-Mannsberger, S., Bakonyi, T., Weissenbock, H., and Nowotny, N. Explaining Usutu virus dynamics in Austria: model development and calibration. Preventive Veterinary Medicine. 85(2008): 166-186.

[61] Safi, M.A., Imran, M. and Gumel, A.B. Threshold dynamics of a non-autonomous SEIRS model with quarantine and isolation. Theory in Biosciences. 131(2012): 19-30.

[62] Sinka, M.E. et al. The dominant Anopheles vectors of human malaria in Africa, Europe and the Middle East: occurrence data, distribution maps and bionomic precis. Parasites and Vectors. 3(2010): 117. (doi:10.1186/1756-3305-3-117).

[63] Smith, H.L. Monotone Dynamical Systems: an Introduction to the Theory of Competitive and Cooperative Systems. American Mathematical Soc. 41(1995).

[64] South Africa's Population. Statistics South Africa. http://www.southafrica.info/about/people/population.htm. Accessed: June 2015.

[65] Sutherst, R.W. Global change and human vulnerability to vector-borne diseases. Clinical Microbiology Reviews. 17(2004):136-173. 
[66] Suwanchaichinda, C. and Paskewitz, S.M. 1998 Effects of larval nutrition, adult body size, and adult temperature on the ability of Anopheles gambiae (Diptera: Culicidae) to melanize sephadex beads. Journal of Medical Entomology. 35(1998): 157-161.

[67] Tabachnick, W.J. Challenges in predicting climate and environmental effects on vectorborne disease episystems in a changing world. The Journal of Experimental Biology. 213(2010): 946-954.

[68] Thieme, H.R. Convergence results and a Poincaré-Bendixson trichotomy for asymptotically autonomous differential equations. Journal of Mathematical Biology. 30(1992): 755-763.

[69] Thieme, H.R. Persistence under relaxed point dissipativity (with application to an endemic model). SIAM Journal on Mathematical Analysis. 24(1993): 407-411.

[70] United Nations, Department of Economic and Social Affairs, Population Division. World Population Prospects: The 2010 Revision. 2011.

http://www.un.org/en/. Accessed: July 2015.

[71] Van den Driessche, P. and Watmough, J. Reproduction numbers and sub-threshold endemic equilibria for compartmental models of disease transmission. Mathematical Biosciences. 180(2002): 29-48.

[72] Wang, W. and Zhao, X.-Q. Threshold dynamics for compartmental epidemic models in periodic environments. Journal of Dynamics and Differential Equations. 20(2008): 699-717.

[73] World Health Organization. World Malaria Report (2008). http://www.who.int/malaria/publications/atoz/9789241563697/en/. Accessed: June 2015.

[74] Yang, H.M., Macoris, M.L., Galvani, K.C., Andrighetti, M.T. and Wanderley, D.M. Assessing the effects of temperature on dengue transmission. Epidemiology and Infection. 137(2009): 1179-1187. (doi:10.1017/S0950268809002052).

[75] Yang, H.M., Macoris, M.L., Galvani, K.C., Andrighetti, M.T., Wanderley, D.M. Assessing the effects of temperature on the population of Aedes aegypti, the vector of dengue. Epidemiology and Infection. 137(2009): 1188-1202. (doi:10.1017/S0950268809002040).

[76] Zhang, F. and Zhao, X.-Q. A periodic epidemic model in a patchy environment. Journal of Mathematical Analysis and Applications. 325(2007): 496-516.

[77] Zhang, Z., Ding, T.W., Huang, T. and Dong, Z. Qualitative Theory of Differential Equations. American Mathematical. (2006).

[78] Zhao, X.-Q. Uniform persistence and periodic coexistence states in infinite-dimensional periodic semiflows with applications. Canadian Applied Mathematics Quarterly. 3(1995): 473-495. 
[79] Zhao, X.-Q. Dynamical Systems in Population Biology. Springer, New York. (2003).

[80] Zhao, X.-Q. Permanence Implies the Existence of Interior Periodic Solutions for FDEs. International Journal of Qualitative Theory of Differential Equations and Applications 2(2008): 125-137. 


\section{Appendix A: Proof of Theorem 3.4}

Proof. Consider the special case of the autonomous version of the model (2.1) with $\eta=$ $\delta_{c}=\delta_{a}=\delta_{c r}=\delta_{a r}=0$. It follows, by substituting $\eta=\delta_{c}=\delta_{a}=\delta_{c r}=\delta_{a r}=0$ into the autonomous version of the model $(2.1)$, that $N_{h}^{*}=\Pi_{c} / \mu_{h}$. Furthermore, let $\mathcal{R}_{0 *}<1$. Since all solutions of the autonomous version of the model remain in $C\left([0], \mathbb{R}_{+}^{19}\right) \backslash\left\{\mathcal{T}_{0}\right\}$, it follows that:

$$
\begin{aligned}
M_{a}(t) & \leq\left(1-\frac{1}{\mathcal{N}}\right) K_{v}, M_{s}(t) \leq \frac{\xi_{l}}{\mu_{m}}\left(1-\frac{1}{\mathcal{N}}\right) K_{v} \\
S_{c}(t) & \leq \frac{\Pi_{c}}{\mu_{h}+\xi_{h}} \text { and } S_{a}(t) \leq \frac{\xi_{h} \Pi_{c}}{\mu_{h}\left(\mu_{h}+\xi_{h}\right)}
\end{aligned}
$$

Thus, the equations for the rate of change of the infected variables (of the special case of the autonomous version of the model (2.1)) can be re-written as:

$$
\begin{aligned}
\frac{d E_{c}(t)}{d t} & \leq \frac{b_{m} \mu_{h} \beta_{c} M_{i}}{\mu_{h}+\xi_{h}}-\left(\sigma_{c}+\mu_{h}\right) E_{c}, \\
\frac{d I_{c}(t)}{d t} & =\sigma_{c} E_{c}-\left(\gamma_{c}+\mu_{h}\right) I_{c} \\
\frac{d E_{a}(t)}{d t} & \leq \frac{b_{m} \xi_{h} \beta_{a} M_{i}}{\mu_{h}+\xi_{h}}-\left(\sigma_{a}+\mu_{h}\right) E_{a}, \\
\frac{d I_{a}(t)}{d t} & =\sigma_{a} E_{a}-\left(\gamma_{a}+\mu_{h}\right) I_{a}, \\
\frac{d M_{i}(t)}{d t} & \leq \frac{b_{m} \beta_{m}\left(I_{c}+I_{a}\right) \mu_{h} \xi_{l} M_{a}^{*}}{\mu_{m} \Pi_{c}}-\mu_{m} M_{i} .
\end{aligned}
$$

Using a standard comparison theorem [34], it only suffices to show that every (upper) solution of the system

$$
\begin{aligned}
\frac{d E_{c}(t)}{d t} & =\frac{b_{m} \mu_{h} \beta_{c} M_{i}}{\mu_{h}+\xi_{h}}-\left(\sigma_{c}+\mu_{h}\right) E_{c} \\
\frac{d I_{c}(t)}{d t} & =\sigma_{c} E_{c}-\left(\gamma_{c}+\mu_{h}\right) I_{c} \\
\frac{d E_{a}(t)}{d t} & =\frac{b_{m} \xi_{l} \beta_{a} M_{i}}{\mu_{h}+\xi_{h}}-\left(\sigma_{a}+\mu_{h}\right) E_{a} \\
\frac{d I_{a}(t)}{d t} & =\sigma_{a} E_{a}-\left(\gamma_{a}+\mu_{h}\right) I_{a} \\
\frac{d M_{i}(t)}{d t} & =\frac{b_{m} \beta_{m}\left(I_{c}+I_{a}\right) \mu_{h} \xi_{l} M_{a}^{*}}{\mu_{m} \Pi_{c}}-\mu_{m} M_{i}
\end{aligned}
$$

converges to zero as $t \rightarrow \infty$. It can be shown that the system (A-3) is cooperative and irreducible, with a unique equilibrium in the closure of the open set [63]

$$
\mathcal{D}=\left\{\left(E_{c}, I_{c}, E_{a}, I_{a}, M_{i}\right): E_{c}>0, I_{c}>0, E_{a}>0, I_{a}>0, M_{i}>0\right\}
$$


Thus, it follows, from Theorem 3.1 in [63], that

$$
\left(E_{c}(t), I_{c}(t), E_{a}(t), I_{a}(t), M_{i}(t)\right) \rightarrow(0,0,0,0,0), \text { as } t \rightarrow \infty .
$$

Since $\left(I_{c}(t), I_{a}(t), M_{i}(t)\right) \rightarrow(0,0,0)$ as $t \rightarrow \infty$, it follows that $I_{c}(t)<\epsilon_{1}, I_{a}(t)<\epsilon_{2}$ and $M_{i}<\epsilon_{3}$ for large $t$ and sufficiently small $\epsilon_{1}, \epsilon_{2}$ and $\epsilon_{3}$. Thus, the following inequalities hold

$$
\begin{aligned}
& \frac{d S_{c}(t)}{d t}>\Pi_{c}-\frac{b_{m} \mu_{h} \beta_{c} \epsilon_{3}}{\Pi_{c}} S_{c}-\left(\mu_{h}+\xi_{h}\right) S_{c}, \\
& \frac{d S_{a}(t)}{d t}>\xi_{h} S_{c}-\frac{b_{m} \beta_{a} \mu_{h} \epsilon_{3}}{\Pi_{c}} S_{a}-\mu_{h} S_{a}, \\
& \frac{d M_{a}(t)}{d t}>\alpha_{l}\left(1-\frac{M_{a}}{K_{v}}\right)\left(M_{s}+\epsilon_{3}\right)-\left(\xi_{l}+\mu_{l}\right) M_{a}, \\
& \frac{d M_{s}(t)}{d t}>\xi_{l} M_{a}-\frac{b_{m} \mu_{h} \beta_{m}\left(\epsilon_{1}+\epsilon_{2}\right)}{\Pi_{c}} M_{s}-\mu_{m} M_{s},
\end{aligned}
$$

so that,

$$
\begin{aligned}
\liminf _{t \rightarrow \infty} S_{c}(t) & \geq \frac{\Pi_{c}}{\frac{b_{m} \mu_{h} \beta_{c} \epsilon_{3}}{\Pi_{c}}+\left(\mu_{h}+\xi_{h}\right)}, \liminf _{t \rightarrow \infty} S_{a}(t) \geq \frac{\xi_{h} S_{c}}{\frac{b_{m} \mu_{h} \beta_{c} \epsilon_{3}}{\Pi_{c}}+\mu_{h}} \text { and } \\
\liminf _{t \rightarrow \infty} M_{s}(t) & \geq \frac{\xi_{l} M_{a}}{\frac{b_{m} \mu_{h} \beta_{m}\left(\epsilon_{1}+\epsilon_{2}\right)}{\Pi_{c}}+\mu_{m}} .
\end{aligned}
$$

Letting $\left(\epsilon_{1}, \epsilon_{2}, \epsilon_{3}\right) \rightarrow(0,0,0)$ in $(\mathrm{A}-5)$ gives

$$
\begin{aligned}
\liminf _{t \rightarrow \infty} S_{c}(t) & \geq \frac{\Pi_{c}}{\mu_{h}+\xi_{h}}, \liminf _{t \rightarrow \infty} S_{a}(t) \geq \frac{\xi_{h} \Pi_{c}}{\mu_{h}\left(\mu_{h}+\xi_{h}\right)}, \\
\liminf _{t \rightarrow \infty} M_{a}(t) & \geq\left(1-\frac{1}{\mathcal{N}}\right) K_{v}, \quad \liminf _{t \rightarrow \infty} M_{s}(t) \geq \frac{\xi_{l}}{\mu_{m}}\left(1-\frac{1}{\mathcal{N}}\right) K_{v} .
\end{aligned}
$$

Combining (A-1) and (A-6) shows that, for $\mathcal{R}_{0 *}<1$,

$$
\begin{aligned}
\liminf _{t \rightarrow \infty} S_{c}(t) & =\frac{\Pi_{c}}{\mu_{h}+\xi_{h}}, \liminf _{t \rightarrow \infty} S_{a}(t)=\frac{\xi_{h} \Pi_{c}}{\mu_{h}\left(\mu_{h}+\xi_{h}\right)}, \\
\liminf _{t \rightarrow \infty} M_{a}(t) & =\left(1-\frac{1}{\mathcal{N}}\right) K_{v}, \quad \liminf _{t \rightarrow \infty} M_{s}(t)=\frac{\xi_{l}}{\mu_{m}}\left(1-\frac{1}{\mathcal{N}}\right) K_{v} .
\end{aligned}
$$

Hence, it follows, from (A-4) and (A-7), that for $\mathcal{R}_{0 *}<1$,

$$
\begin{aligned}
& \left(S_{c}(t), E_{c}(t), I_{c}(t), S_{a}(t), E_{a}(t), I_{a}(t), M_{a}(t), M_{s}(t), M_{i}(t)\right) \\
& \rightarrow\left(\frac{\Pi_{c}}{\mu_{h}+\xi_{h}}, 0,0, \frac{\xi_{h} \Pi_{c}}{\mu_{h}\left(\mu_{h}+\xi_{h}\right)}, 0,0,\left(1-\frac{1}{\mathcal{N}}\right) K_{v}, \frac{\xi_{l}}{\mu_{m}}\left(1-\frac{1}{\mathcal{N}}\right) K_{v}, 0\right) \text { as } t \rightarrow \infty .
\end{aligned}
$$

Using (A-8) in the autonomous version of the model (2.1), it can be shown that

$$
\left(S_{c r}, E_{c r}, I_{c r}, S_{a r}, E_{a r}, I_{a r}, W_{c}, W_{c r}, W_{a}, W_{a r}\right)(t) \rightarrow(0,0,0,0,0,0,0,0,0,0) \text { as } t \rightarrow \infty .
$$

Thus, by combining (A-8) and (A-9), it follows that the NDFE $\left(\mathcal{E}_{0}\right)$ of the autonomous version of the model $(2.1)$, with $\eta=\delta_{c}=\delta_{a}=\delta_{c r}=\delta_{a r}=0$, is GAS in $C\left([0], \mathbb{R}_{+}^{19}\right) \backslash\left\{\mathcal{T}_{0}\right\}$ whenever $\mathcal{R}_{0 *}<1$. 


\section{Appendix B: Proof of Theorem 3.5}

Proof. Consider the special case of the autonomous version of model (2.1) with $\eta=\delta_{c}=\delta_{a}=$ $\delta_{c r}=\delta_{a r}=0$ and $\mathcal{R}_{0 *}>1$ (so that the unique endemic equilibrium exists in line with Theorem 3.3(i)). Let $\mathcal{E}_{1}=\left(S_{c}^{* *}, S_{c r}^{* *}, E_{c}^{* *}, E_{c r}^{* *}, I_{c}^{* *}, I_{c r}^{* *}, W_{c}^{* *}, W_{c r}^{* *}, S_{a}^{* *}, S_{a r}^{* *}, E_{a}^{* *}, E_{a r}^{* *}, I_{a}^{* *}, I_{a r}^{* *}, W_{a}^{* *}\right.$, $\left.W_{a r}^{* *}, M_{a}^{* *}, M_{s}^{* *}, M_{i}^{* *}\right)$ represents this unique endemic equilibrium. Consider, further, the following non-linear Lyapunov function of Goh-Volterra type:

$$
\begin{aligned}
\mathcal{K}= & S_{c}-S_{c}^{* *} \ln S_{c}+S_{c r}-S_{c r}^{* *} \ln S_{c r}+E_{c}-E_{c}^{* *} \ln E_{c}+E_{c r}-E_{c r}^{* *} \ln E_{c r}+d_{1}\left(I_{c}-I_{c}^{* *} \ln I_{c}\right) \\
& +d_{2}\left(I_{c r}-I_{c r}^{* *} \ln I_{c r}\right)+d_{3}\left(W_{c}-W_{c}^{* *} \ln W_{c}\right)+d_{4}\left(W_{c r}-W_{c r}^{* *} \ln W_{c r}\right)+S_{a}-S_{a}^{* *} \ln S_{a}+S_{a r} \\
& -S_{a r}^{* *} \ln S_{a r}+E_{a}-E_{a}^{* *} \ln E_{a}+E_{a r}-E_{a r}^{* *} \ln E_{a r}+d_{5}\left(I_{a}-I_{a}^{* *} \ln I_{a}\right)+d_{6}\left(I_{a r}-I_{a r}^{* *} \ln I_{a r}\right) \\
& +d_{7}\left(W_{a}-W_{a}^{* *} \ln W_{a}\right)+d_{8}\left(W_{a r}-W_{a r}^{* *} \ln W_{a r}\right)+M_{a}-M_{a}^{* *} \ln M_{a}+M_{s}-M_{s}^{* *} \ln M_{s} \\
& +M_{i}-M_{i}^{* *} \ln M_{i},
\end{aligned}
$$

where,

$d_{1}=\frac{k_{3}}{\sigma_{c}}, d_{2}=\frac{k_{4}}{\sigma_{c r}}, d_{3}=\frac{k_{3} k_{5}}{\sigma_{c} \gamma_{c}}, d_{4}=\frac{k_{4} k_{5}}{\sigma_{c r} \gamma_{c r}}, d_{5}=\frac{k_{9}}{\sigma_{a}}, d_{6}=\frac{k_{10}}{\sigma_{a r}}, d_{7}=\frac{k_{9} k_{11}}{\sigma_{a} \gamma_{a}}, d_{8}=\frac{k_{10} k_{12}}{\sigma_{a r} \gamma_{a r}}$.

It is convenient to define the following steady-state relations (obtained from solving the aforementioned special case of the autonomous version of the model (2.1) at the endemic steady-state, $\left.\mathcal{E}_{1}\right)$ :

$$
\begin{aligned}
& \Pi_{c}=\lambda_{c}^{* *} S_{c}^{* *}+k_{1} S_{c}^{* *}, \psi_{c}\left(W_{c}^{* *}+\theta_{c r} W_{c r}^{* *}\right)=\lambda_{c r}^{* *} S_{c r}^{* *}+k_{2} S_{c r}^{* *}, \lambda_{c}^{* *} S_{c}^{* *}=k_{3} E_{c}^{* *}, \lambda_{c r}^{* *} S_{c r}^{* *}=k_{4} E_{c r}^{* *}, \\
& \sigma_{c} E_{c}^{* *}=k_{5} I_{c}^{* *}, \sigma_{c r} E_{c r}^{* *}=k_{6} I_{c r}^{* *}, \gamma_{c} I_{c}^{* *}=k_{7} W_{c}^{* *}, \gamma_{c r} I_{c r}^{* *}=k_{8} W_{c r}^{* *}, \xi_{h} S_{c}^{* *}=\lambda_{a}^{* *} S_{a}+\mu_{h} S_{a}^{* *}, \\
& \psi_{a}\left(W_{a}^{* *}+\theta_{a r} W_{a r}^{* *}\right)+\xi_{h} S_{c r}^{* *}=\lambda_{a r}^{* *} S_{a r}^{* *}+\mu_{h} S_{a r}^{* *}, \lambda_{a}^{* *} S_{a}^{* *}=k_{9} E_{a}^{* *}, \lambda_{a r}^{* *} S_{a r}^{* *}=k_{10} E_{a r}^{* *}, \\
& \sigma_{a} E_{a}^{* *}=k_{11} I_{a}^{* *}, \sigma_{a r} E_{a r}^{* *}=k_{12} I_{a r}^{* *}, \gamma_{a} I_{a}^{* *}=k_{13} W_{a}^{* *}, \gamma_{a r} I_{a r}^{* *}=k_{14} W_{a r}^{* *}, \lambda_{m}^{* *} M_{s}^{* *}=\mu_{m} M_{i}^{* *}, \\
& \alpha_{l}\left(1-\frac{M_{a}^{* *}}{K_{v}}\right)\left(M_{s}^{* *}+M_{i}^{* *}\right)=\xi_{l} M_{a}^{* *}+\mu_{l} M_{a}^{* *}, \xi_{l} M_{a}^{* *}=\lambda_{m}^{* *} M_{s}^{* *}+\mu_{m} M_{s}^{* *},
\end{aligned}
$$

with,

$$
\begin{aligned}
& k_{1}=k_{2}=\xi_{h}+\mu_{h}, k_{3}=\sigma_{c}+\mu_{h}, k_{4}=\sigma_{c r}+\mu_{h}, k_{5}=\gamma_{c}+\mu_{h}, k_{6}=\gamma_{c r}+\mu_{h}, k_{7}=\mu_{h}+\psi_{c}, \\
& k_{8}=\mu_{h}+\theta_{c r} \psi_{c}, k_{9}=\sigma_{a}+\mu_{h}, k_{10}=\sigma_{a r}+\mu_{h}, k_{11}=\gamma_{a}+\mu_{h}, k_{12}=\gamma_{a r}+\mu_{h}, k_{13}=\mu_{h}+\psi_{a}, \\
& k_{14}=\mu_{h}+\theta_{a r} \psi_{a}, \lambda_{c}^{* *}=\frac{b_{m} \beta_{c} \mu_{h} M_{i}^{* *}}{\prod_{c}}, \lambda_{a}^{* *}=\frac{b_{m} \beta_{a} \mu_{h} M_{i}^{* *}}{\Pi_{c}}, \lambda_{m}^{* *}=\frac{b_{m} \beta_{m} \mu_{h}\left[I_{a}^{* *}+I_{c}^{* *}\right]}{\Pi_{c}}, \\
& \lambda_{c r}^{* *}=\left(1-\epsilon_{c}\right) \lambda_{c}^{* *} \text { and } \lambda_{a r}^{* *}=\left(1-\epsilon_{a}\right) \lambda_{a}^{* *} .
\end{aligned}
$$


The Lyapunov derivative of $\mathcal{K}$ is

$$
\begin{aligned}
\mathcal{K}^{\prime}= & \left(1-\frac{S_{c}^{* *}}{S_{c}}\right)\left(\Pi_{c}-\lambda_{c} S_{c}-k_{1} S_{c}\right)+\left(1-\frac{S_{c r}^{* *}}{S_{c r}}\right)\left[\psi_{c}\left(W_{c}+\theta_{c r} W_{c r}\right)-\left(1-\epsilon_{c}\right) \lambda_{c} S_{c r}-k_{2} S_{c r}\right] \\
& +\left(1-\frac{E_{c}^{* *}}{E_{c}}\right)\left(\lambda_{c} S_{c}-k_{3} E_{c}\right)+\left(1-\frac{E_{c r}^{* *}}{E_{c r}}\right)\left[\left(1-\epsilon_{c}\right) \lambda_{c} S_{c r}-k_{4} E_{c r}\right]+d_{1}\left(1-\frac{I_{c}^{* *}}{I_{c}}\right)\left(\sigma_{c} E_{c}\right. \\
& \left.-k_{5} I_{c}\right)+d_{2}\left(1-\frac{I_{c r}^{* *}}{I_{c r}}\right)\left(\sigma_{c r} E_{c r}-k_{6} I_{c r}\right)+d_{3}\left(1-\frac{R_{c}^{* *}}{R_{c}}\right)\left(\gamma_{c} I_{c}-k_{7} W_{c}\right)+d_{4}\left(1-\frac{W_{c r}^{* *}}{W_{c r}}\right) \\
& \left(\gamma_{c r} I_{c r}-k_{8} W_{c r}\right)+\left(1-\frac{S_{a}^{* *}}{S_{a}}\right)\left(\xi_{h} S_{c}-\lambda_{a} S_{a}-\mu_{h} S_{a}\right)+\left(1-\frac{S_{a r}^{* *}}{S_{a r}}\right)\left[\psi_{a}\left(W_{a}+\theta_{a r} W_{a r}\right)\right. \\
& \left.+\xi_{h} S_{c r}-\left(1-\epsilon_{a}\right) \lambda_{a} S_{a r}-\mu_{h} S_{a r}\right]+\left(1-\frac{E_{a}^{* *}}{E_{a}}\right)\left(\lambda_{a} S_{a}-k_{9} E_{a}\right)+\left(1-\frac{E_{a r}^{* *}}{E_{a r}}\right)\left[\left(1-\epsilon_{a}\right)\right. \\
& \left.\lambda_{a} S_{a r}-k_{10} E_{a r}\right]+d_{5}\left(1-\frac{I_{a}^{* *}}{I_{a}}\right)\left(\sigma_{a} E_{a}-k_{11} I_{a}\right)+d_{6}\left(1-\frac{I_{a r}^{* *}}{I_{a r}}\right)\left(\sigma_{a r} E_{a r}-k_{12} I_{a r}\right) \\
& +d_{7}\left(1-\frac{W_{a}^{* *}}{W_{a}}\right)\left(\gamma_{a} I_{a}-k_{13} W_{a}\right)+d_{8}\left(1-\frac{W_{a r}^{* *}}{W_{a r}}\right)\left(\gamma_{a r} I_{a r}-k_{14} W_{a r}\right)+\left(1-\frac{M_{a}^{* *}}{M_{a}}\right) \\
& {\left[\alpha_{l}\left(1-\frac{M_{a}}{K_{v}}\right)\left(M_{s}+M_{i}\right)-\xi_{l} M_{a}-\mu_{l} M_{a}\right]+\left(1-\frac{M_{s}^{* *}}{M_{s}}\right)\left(\xi_{l} M_{a}-\lambda_{m} M_{s}-\mu_{m} M_{s}\right) } \\
& +\left(1-\frac{M_{s}^{* *}}{M_{s}}\right)\left(\lambda_{m} M_{s}-\mu_{m} M_{i}\right) .
\end{aligned}
$$

Using the steady-state relations (B-1) in (B-2), and noting that the state variables of the autonomous version of the model (2.1) are bounded by their endemic steady-state values in $\Omega$ (Theorem 3.5 ), gives (in $\Omega$ )

$$
\begin{aligned}
\mathcal{K}^{\prime} \leq & \left(1-\frac{S_{c}^{* *}}{S_{c}}\right)\left(\lambda_{c}^{* *} S_{c}^{* *}+k_{1} S_{c}^{* *}-k_{1} S_{c}\right)+\lambda_{c}^{* *} S_{c}^{* *}+\left(1-\frac{S_{c r}^{* *}}{S_{c r}}\right)\left(\lambda_{c r}^{* *} S_{c r}^{* *}+k_{2} S_{c r}^{* *}-k_{2} S_{c r}\right) \\
& +\lambda_{c r}^{* *} S_{c r}^{* *}-\frac{E_{c}^{* *}}{E_{c}}\left(\lambda_{c}^{* *} S_{c}-k_{3} E_{c}\right)-\frac{E_{c r}^{* *}}{E_{c r}}\left(\lambda_{c r}^{* *} S_{c r}-k_{4} E_{c r}\right)-d_{1} \frac{I_{c}^{* *}}{I_{c}}\left(\sigma_{c} E_{c}-k_{5} I_{c}\right)-d_{2} \frac{I_{c r}^{* *}}{I_{c r}} \\
& \left(\sigma_{c r} E_{c r}-k_{6} I_{c r}\right)-d_{3} \frac{W_{c}^{* *}}{W_{c}}\left(\gamma_{c} I_{c}-k_{7} W_{c}\right)-d_{4} \frac{W_{c r}^{* *}}{W_{c r}}\left(\gamma_{c r} I_{c r}-k_{8} W_{c r}\right)+\left(1-\frac{S_{a}^{* *}}{S_{a}}\right)\left(\xi_{h} S_{c}^{* *}\right. \\
& \left.-\mu_{h} S_{a}\right)+\lambda_{a}^{* *} S_{a}^{* *}+\left(1-\frac{S_{a r}^{* *}}{S_{a r}}\right)\left(\lambda_{a r}^{* *} S_{a r}^{* *}+\mu_{h} S_{a r}^{* *}-\mu_{h} S_{a r}\right)+\lambda_{a}^{* *} S_{a}^{* *}-\frac{E_{a}^{* *}}{E_{a}}\left(\lambda_{a}^{* *} S_{a}\right. \\
& \left.-k_{9} E_{a}\right)-\frac{E_{a r}^{* *}}{E_{a r}}\left(\lambda_{a r}^{* *} S_{a r}-k_{10} E_{a r}\right)-d_{5} \frac{I_{a}^{* *}}{I_{a}}\left(\sigma_{a} E_{a}-k_{11} I_{a}\right)-d_{6} \frac{I_{a r}^{* *}}{I_{a r}}\left(\sigma_{a r} E_{a r}-k_{12} I_{a r}\right) \\
& -d_{7} \frac{W_{a}^{* *}}{W_{a}}\left(\gamma_{a} I_{a}-k_{13} R_{a}\right)-\frac{W_{a r}^{* *}}{W_{a r}}\left(\gamma_{a r} I_{a r}-k_{14} R_{a r}\right)+\left(1-\frac{M_{a}^{* *}}{M_{a}}\right)\left(\xi_{l} M_{a}^{* *}+\mu_{l} M_{a}^{* *}\right. \\
& \left.-\mu_{l} M_{a}\right)+\xi_{l} M_{a}^{* *}-\mu_{m} M_{s}-\frac{M_{s}^{* *}}{M_{s}}\left(\lambda_{m}^{* *} M_{s}^{*}+\mu_{m} M_{s}^{* *}-\lambda_{m} M_{s}-\mu_{m} M_{s}\right)-\mu_{m} M_{i} \\
& -\frac{M_{i}^{* *}}{M_{i}}\left(\mu_{m} M_{i}^{* *}-\mu_{m} M_{i}\right),
\end{aligned}
$$


which can further be simplified to

$$
\begin{aligned}
\mathcal{K}^{\prime} \leq & -\xi_{l} \frac{M_{a}^{* *}}{M_{a}}\left(M_{a}^{* *}-M_{a}\right)-\frac{M_{s}^{* *}}{M_{s}}\left(\lambda_{m}^{* *} M_{s}^{* *}-\lambda_{m} M_{s}\right)+k_{1} S_{c}^{* *}\left(2-\frac{S_{c}^{* *}}{S_{c}}-\frac{S_{c}}{S_{c}^{* *}}\right)+k_{2} S_{c r}^{* *}(2 \\
& \left.-\frac{S_{c r}^{* *}}{S_{c r}}-\frac{S_{c r}}{S_{c r}^{* *}}\right)+\mu_{h} S_{a}^{* *}\left(2-\frac{S_{a}^{* *}}{S_{a}}-\frac{S_{a}}{S_{a}^{* *}}\right)+\mu_{h} S_{a r}^{* *}\left(2-\frac{S_{a r}^{* *}}{S_{a r}}-\frac{S_{a r}}{S_{a r}^{* *}}\right)+\mu_{l} M_{a}^{* *}\left(2-\frac{M_{a}^{* *}}{M_{a}}\right. \\
& \left.-\frac{M_{a}}{M_{a}^{* *}}\right)+\mu_{m} M_{s}^{* *}\left(2-\frac{M_{s}^{* *}}{M_{s}}-\frac{M_{s}}{M_{s}^{* *}}\right)+\mu_{m} M_{i}^{* *}\left(2-\frac{M_{i}^{* *}}{M_{i}}-\frac{M_{i}}{M_{i}^{* *}}\right)+k_{3} E_{c}^{* *}\left(5-\frac{S_{c}^{* *}}{S_{c}}\right. \\
& \left.-\frac{W_{c}}{W_{c}^{* *}}-\frac{I_{c} W_{c}^{* *}}{I_{c}^{* *} W_{c}}-\frac{I_{c}^{* *} E_{c}}{I_{c} E_{c}^{* *}}-\frac{S_{c} E_{c}^{* *}}{S_{c}^{* *} E_{c}}\right)+k_{4} E_{c r}^{* *}\left(5-\frac{S_{c r}^{* *}}{S_{c r}}-\frac{W_{c r}}{W_{c r}^{* *}}-\frac{I_{c r} W_{c r}^{* *}}{I_{c r}^{* *} W_{c r}}-\frac{I_{c r}^{* *} E_{c r}}{I_{c r} E_{c r}^{* *}}\right. \\
& \left.-\frac{S_{c r} E_{c r}^{* *}}{S_{c r}^{* *} E_{c r}}\right)+k_{9} E_{a}^{* *}\left(5-\frac{S_{a}^{* *}}{S_{a}}-\frac{W_{a}}{W_{a}^{* *}}-\frac{I_{a} W_{a}^{* *}}{I_{a}^{* *} W_{a}}-\frac{I_{a}^{* *} E_{a}}{I_{a} E_{a}^{* *}}-\frac{S_{a} E_{a}^{* *}}{S_{a}^{* *} E_{a}}\right)+k_{10} E_{a r}^{* *}\left(5-\frac{S_{a r}^{* *}}{S_{a r}}\right. \\
& \left.-\frac{W_{a r}}{W_{a r}^{* *}}-\frac{I_{a r} W_{a r}^{* *}}{I_{a r}^{* *} W_{a r}}-\frac{I_{a r}^{* *} E_{a r}}{I_{a r} E_{a r}^{* *}}-\frac{S_{a r} E_{a r}^{* *}}{S_{a r}^{* *} E_{a r}}\right) .
\end{aligned}
$$

The first two terms of (B-3) are automatically negative in $\Omega$. Furthermore, since the arithmetic mean exceeds the geometric mean, it follows that the remaining terms of (B-3) are also negative. Hence, $\mathcal{K}^{\prime} \leq 0$. The proof is concluded as in the proof of Theorem 3.1. Thus, the unique endemic equilibrium, $\mathcal{E}_{1}$, of the autonomous model with $\eta=\delta_{c}=\delta_{a}=\delta_{c r}=\delta_{a r}=0$, is GAS in $\Omega_{1}$ whenever $\mathcal{R}_{0 *}>1$.

\section{Appendix C: Proof of Theorem 4.2}

Proof. Consider the non-autonomous model (2.1) with $\delta_{c}=\delta_{a}=\delta_{c r}=\delta_{a r}=0 . \mathcal{N}_{n}(t)>1$ for all $t \geq 0$. Furthermore, let $\mathcal{R}_{0 T R *}<1$. As in Appendix A, the proof is based on using a comparison theorem [63]. Using the fact that $S_{c}(t) \leq \frac{\Pi_{c}}{\mu_{h}+\xi_{h}}, S_{a}(t) \leq \frac{\xi_{h} \Pi_{c}}{\mu_{h}\left(\mu_{h}+\xi_{h}\right)}$, $M_{a}(t) \leq\left(1-\frac{1}{\mathcal{N}_{n}(t)}\right) K_{v}$ and $M_{s}(t) \leq \frac{\xi_{l}(t)}{\mu_{m}(t)}\left(1-\frac{1}{\mathcal{N}_{n}(t)}\right) K_{v}$ for all $t \geq 0$ in $C\left([0], \mathbb{R}_{+}^{19}\right) \backslash\left\{\mathcal{T}_{0}\right\}$, it follows that the non-autonomous model (2.1), subject to the aforementioned assumptions, 
can be re-written as:

$$
\begin{aligned}
\frac{d E_{c}}{d t} & \leq \frac{b_{m}(t) \beta_{c} \mu_{h} M_{i}}{\mu_{h}+\xi_{h}}-\left(\sigma_{c}+\mu_{h}\right) E_{c}, \\
\frac{d E_{c r}}{d t} & =\left(1-\epsilon_{c}\right) \frac{b_{m}(t) \beta_{c} \mu_{h} M_{i}}{\Pi_{c}} S_{c r}-\left(\sigma_{c r}+\mu_{h}\right) E_{c r}, \\
\frac{d I_{c}}{d t} & =\sigma_{c} E_{c}-\left(\gamma_{c}+\mu_{h}\right) I_{c}, \\
\frac{d I_{c r}}{d t} & =\sigma_{c r} E_{c r}-\left(\gamma_{c r}+\mu_{h}\right) I_{c r}, \\
\frac{d E_{a}}{d t} & \leq \frac{b_{m}(t) \beta_{a} \xi_{h} M_{i}}{\mu_{h}+\xi_{h}}-\left(\sigma_{a}+\mu_{h}\right) E_{a}, \\
\frac{d E_{a r}}{d t} & =\left(1-\epsilon_{a}\right) \frac{b_{m}(t) \beta_{a} \mu_{h} M_{i}}{\Pi_{c}} S_{a r}-\left(\sigma_{a r}+\mu_{h}\right) E_{a r}, \\
\frac{d I_{a}}{d t} & =\sigma_{a} E_{a}-\left(\gamma_{a}+\mu_{h}\right) I_{a}, \\
\frac{d I_{a r}}{d t} & =\sigma_{a r} E_{a r}-\left(\gamma_{a r}+\mu_{h}\right) I_{a r}, \\
\frac{d M_{i}}{d t} & \leq \frac{b_{m}(t) \beta_{m} \mu_{h}\left[I_{a}+I_{c}+\eta\left(I_{c r}+I_{a r}\right)\right] M_{s n}}{\Pi_{c}}-\mu_{m}(t) M_{i} .
\end{aligned}
$$

Following [72], the equations in (C-1), with equalities used in place of the inequalities, can be re-written in terms of the next generation matrices $F(t)$ and $V(t)$, as follows

$$
\frac{d W(t)}{d t}=[F(t)-V(t)] W(t)
$$

It follows, from Lemma 2.1 in [76], that there exists a positive $\tau$-periodic function, $w(t)=$ $\left(\underline{E_{c}}(t), \underline{E_{c r}}(t), \underline{I_{c}}(t), \underline{I_{c r}}(t), \underline{E_{a}}(t), \underline{E_{a r}}(t), \underline{I_{a}}(t), \underline{I_{a r}}(t), \underline{M_{i}}(t)\right)$, such that

$$
W(t)=e^{\theta t} w(t), \text { with } \theta=\frac{1}{\tau} \ln \rho\left[\phi_{F-V}(\tau)\right],
$$

is a solution of the linearized system (C-2). Furthermore, the assumption $\mathcal{R}_{0 T R *}<1$ implies that $\rho\left(\phi_{F-V}(\tau)\right)<1$ (by Theorem 2.2 in [72]). Hence, $\theta$ is a negative constant. Thus, $W(t) \rightarrow 0$ as $t \rightarrow \infty$. Thus, the unique (disease-free) solution of the linear system (C-2), given by $W(t)=0$, is GAS. For any non-negative initial solution $\left(E_{c}(0), E_{c r}(0), I_{c}(0), I_{c r}(0)\right.$, $\left.E_{a}(0), E_{a r}(0), I_{a}(0), I_{a r}(0), M_{i}(0)\right)^{T}$ of the system (C-2), there exists a sufficiently large $M^{*}>$ 0 such that

$$
\left(\left(E_{c}, E_{c r}, I_{c}, I_{c r}, E_{a}, E_{a r}, I_{a}, I_{a r}, M_{i}\right)(0)\right)^{T} \leq M^{*}\left(\left(E_{c}, E_{c r}, I_{c}, I_{c r}, E_{a}, E_{a r}, I_{a}, I_{a r}, M_{i}\right)(0)\right)^{T} .
$$

Thus, it follows, by comparison theorem, that

$$
\left(E_{c}(t), E_{c r}(t), I_{c}(t), I_{c r}(t), E_{a}(t), E_{a r}(t), I_{a}(t), I_{a r}(t), M_{i}(t)\right) \leq M^{*} W(t) \text { for all } t>0,
$$

where $M^{*} W(t)$ is also a solution of (C-2). Hence, $\left(E_{c}(t), E_{c r}(t), I_{c}(t), I_{c r}(t), E_{a}(t), E_{a r}(t), I_{a}(t), I_{a r}(t), M_{i}(t)\right) \rightarrow(0,0,0,0,0,0,0,0,0)$, as $t \rightarrow \infty$. 
Finally, it follows from Theorem 1.2 in [63] that

$$
S_{c}(t) \rightarrow \frac{\Pi_{c}}{\left(\mu_{h}+\xi_{h}\right)}, S_{c r}(t) \rightarrow 0, S_{a}(t) \rightarrow \frac{\xi_{h} \Pi_{c}}{\mu_{h}\left(\mu_{h}+\xi_{h}\right)}, S_{a r}(t) \rightarrow 0, \text { as } t \rightarrow \infty .
$$

and $\left(M_{a}(t), M_{s}(t)\right)$ satisfy $\left(\right.$ for $\mathcal{N}_{n}(t)>1$ for all $\left.t\right)$

$$
\begin{aligned}
\frac{d M_{a}(t)}{d t} & =\alpha_{l}(t)\left[1-\frac{M_{a}(t)}{K_{v}}\right] M_{s}(t)-\xi_{l}(t) M_{a}-\mu_{l}(t) M_{a}(t), \\
\frac{d M_{s}(t)}{d t} & =\xi_{l}(t) M_{a}(t)-\mu_{m}(t) M_{s}(t) .
\end{aligned}
$$

Thus, for $\mathcal{R}_{0 T R *}<1$,

$\left(S_{c}, S_{c r}, E_{c}, E_{c r}, I_{c}, I_{c r}, W_{c}, W_{c r}, S_{a}, S_{a r}, E_{a}, E_{a r}, I_{a}, I_{a r}, W_{a}, W_{a r}, M_{a}, M_{s}, M_{i}\right)(t) \rightarrow \mathcal{E}_{0 N}$ as $t \rightarrow \infty$.

Hence, noting that $\mathcal{E}_{0 N}$ exists for $\mathcal{N}_{n}(t)>1$ for all $t \geq 0$ and is asymptotically-stable when $\mathcal{R}_{0 T R *}<1$ (Theorem 4.1), it follows that $\mathcal{E}_{0 N}$ is globally-attractive in $C\left([0], \mathbb{R}_{+}^{19}\right) \backslash\left\{\mathcal{T}_{0}\right\}$ if $\mathcal{R}_{0 T R *}<1[61]$.

\section{Appendix D: Proof of Theorem 4.3}

Proof. Consider the model (2.1) with $\mathcal{R}_{0 T R}>1$ and $\mathcal{N}_{n}(t)>1$ for all $t \geq 0\left(\mathcal{R}_{0 T R}>1\right.$ is needed to ensure that the disease free solution, $\mathcal{E}_{0 N}$, is unstable (by Theorem 4.1), and $\mathcal{N}_{n}(t)>1$ for all $t \geq 0$ is needed to ensure that the mosquito population does not vanish (by Section 4)). The proof is based on using the technique in [36].

Define the set (where the sets $X$ and $X_{0}$ are as defined in Section 4.2),

$\partial X_{0}=X \backslash X_{0}=\left\{\phi \in X: \phi_{i}(0)=0\right.$ for some $\left.i \in\{2,3,4,5,6,7,8,10,11,12,13,14,15,16,19\}\right\}$.

Let $u(t, \phi)$ be the unique solution of the model (2.1) (through the initial data $u(0, \phi)=\phi$ ). Define $\Phi(t) \varphi=u(t, \varphi)$, the flow generated by the model (2.1) with respect to $\varphi$.

Let $\mathcal{P}: X \rightarrow X$ be the Poincaré map associated with the model (2.1). That is, $\mathcal{P}(\phi)=$ $u(\tau, \phi)$ for all $\phi \in X[36]$. Then, using similar approach as in the proof of Lemma 2.1, it can be shown that $X_{0}$ is a positively-invariant compact set with respect to the model (2.1). Hence, since the solutions of the model (2.1) are uniformly (ultimately)-bounded (Lemma 2.1), it follows that $\mathcal{P}$ is point-dissipative [36]. Furthermore, it follows from Theorem 1.1.2 in [79] that $\mathcal{P}$ admits a global attractor in $\mathrm{X}$.

The next step is to show that $\mathcal{P}$ is uniformly-persistent with respect to $\left(X_{0}, \partial X_{0}\right)[36]$. It is convenient to define (where $\mathcal{P}^{n}(\phi), n \geq 0$, are the periodic points of the Poincaré map) [36]:

$$
\begin{aligned}
K_{\partial} & =\left\{\phi \in \partial X_{0}: \mathcal{P}^{n}(\phi) \in \partial X_{0}, n \geq 0\right\}, \\
D_{1} & =\left\{\phi \in X: \phi_{i}(0)=0 \text { for all } i \in\{2,3,4,5,6,7,8,10,11,12,13,14,15,16,19\}\right\}, \\
D_{2} & =\left\{\phi \in X: \phi_{i}(0)=0 \text { for all } i=17,18,19\right\} .
\end{aligned}
$$


It follows from (D-1) that

$$
\begin{aligned}
D_{1} \cup D_{2}= & \left\{\phi \in X: \phi_{i}(0)=0 \text { for all } i \in\{2,3,4,5,6,7,8,10,11,12,13,14,15,16,\right. \\
& \left.\left.19, \text { or } \phi_{i}(0)=0 \text { for all } i=17,18,19\right\}\right\}, \\
\partial X_{0} \backslash\left(D_{1} \cup D_{2}\right)= & \left\{\phi \in X: \phi_{i}(0) \geq 0 \text { for some } i \in\{2,3,4,5,6,7,8,10,11,12,13,14,15,\right. \\
& 16,19\}\} .
\end{aligned}
$$

In the case where $\mathbf{0}$ is a solution of Equation (2.14), for the total mosquito population $\left(N_{v}^{*}(t)\right)$, we claim the following:

Lemma D.2. $K_{\partial}=D_{1} \cup D_{2}$.

Proof. We first prove that $\left(D_{1} \cup D_{2}\right) \subset K_{\partial}$. It can be seen that for any $\varphi \in D_{2}$, the solution $u_{i}(t, \varphi)=0$ for all $i=17,18,19$. Hence, $D_{2} \subset K_{\partial}$. Similarly, following [36], for any $\varphi \in D_{1}$, we can define a function $G(t) \in C\left(\mathbb{R}_{+}, \mathbb{R}_{+}^{19}\right)$ such that $G_{i}(t) \equiv 0$ for all $t \geq 0$ for $i \in\{2,3,4,5,6,7,8,10,11,12,13,14,15,16,19\}$. Let $G_{1}(t), G_{9}(t), G_{17}(t)$ and $G_{18}(t)$ satisfy the following equations (with $G_{17}(t)<K_{v}$ for all $t$ ), respectively (these are the equations for $S_{c}(t), S_{a}(t), M_{a}(t)$ and $M_{s}(t)$ in the model $\left.(2.1)\right)$ :

$$
\begin{aligned}
& \frac{d G_{1}(t)}{d t}=\Pi_{c}-\left(\xi_{h}+\mu_{h}\right) G_{1}(t) \text { for } t \geq 0 \text { with } G_{1}(0)=\varphi_{1}(0), \\
& \frac{d G_{9}(t)}{d t}=\xi_{h} G_{1}(t)-\mu_{h} G_{9}(t) \text { for } t \geq 0 \text { with } G_{9}(0)=\varphi_{9}(0), \\
& \frac{d G_{17}(t)}{d t}=\alpha_{l}(t)\left(1-\frac{G_{17}(t)}{K_{v}}\right) G_{18}(t)-\left[\xi_{l}(t)+\mu_{l}(t)\right] G_{17}(t) \text { for } t \geq 0 \text { with } G_{17}(0)=\varphi_{17}(0), \\
& \frac{d G_{18}(t)}{d t}=\xi_{l}(t) G_{17}(t)-\mu_{m}(t) G_{18}(t) \text { for } t \geq 0 \text { with } G_{18}(0)=\varphi_{18}(0) .
\end{aligned}
$$

Hence, $G(t)$ is a solution of the model (2.1) through $\varphi$. Furthermore, it follows, by the uniqueness of the solution of the non-autonomous model (Lemma 2.1), that $G(t)=u(t, \varphi)$ for all $t \geq 0$. Hence, $D_{1} \subset K_{\partial}$. Thus, $\left(D_{1} \cup D_{2}\right) \subset K_{\partial}$. To complete the proof of Lemma D.2, we need to show that the converse is true. That is, $K_{\partial} \subset\left(D_{1} \cup D_{2}\right)$. It follows from (D-2) that for any $\varphi \in \partial X_{0} \backslash\left(D_{1} \cup D_{2}\right)$, clearly $\sum_{j=17}^{19} \varphi_{j}(0)>0$. Hence, $\lim _{t \rightarrow \infty}\left|\sum_{j=17}^{19} u_{j}(t, \varphi)-N_{v}^{*}(t)\right|=0$. Let $(2.1)_{k}$ represent the $k$-th equation in the model $(2.1)$. It then follows from $(2.1)_{17}$ and $(2.1)_{18}$ that there exists a $t_{0}>0$ such that $u_{17}(t, \varphi)>0$ and $u_{18}(t, \varphi)>0$ for all $t>t_{0}$. Furthermore, it follows from $(2.1)_{1}$ and $(2.1)_{9}$ that $u_{1}(t, \varphi)>0$ and $u_{9}(t, \varphi)>0$ for all $t>0$. It then follows from $(2.1)_{19}$, noting that $u_{18}(t, \varphi)>0$ for all $t>t_{0}$, that if $\varphi_{19}(0)>0$, then $u_{19}(t, \varphi)>0$ and $t>0$. Furthermore, if $\varphi_{19}(0)>0$, then it ca easily be seen that $u_{j}(t, \varphi)>0$ for all $j \in\{2,3,4,5,6,7,8,10,11,12,13,14,15,16\}$ for all $t>0$. Moreover, it can also be shown that if $\varphi_{3}(0)>0, \varphi_{4}(0)>0, \varphi_{5}(0)>0, \varphi_{6}(0)>0, \varphi_{11}(0)>0$, $\varphi_{12}(0)>0, \varphi_{13}(0)>0$ or $\varphi_{14}(0)>0$, then $u_{j}(t, \varphi)>0$ for all $j \in\{5,6,7,8,13,14,15,16,19\}$ for all $t>0$. If $\varphi_{7}(0)>0$ or $\varphi_{8}(0)>0$, then $(2.1)_{2}$ imply that $u_{2}(t, \varphi)>0$. Similarly, if $\varphi_{15}(0)>0$ or $\varphi_{16}(0)>0$, then $(2.1)_{10}$ imply that $u_{10}(t, \varphi)>0$. Furthermore, if $\varphi_{2}(0)>0$, then $u_{2}(t, \varphi)>0$ and $u_{10}(t, \varphi)>0$ from $(2.1)_{2}$ and $(2.1)_{10}$, respectively. If $\varphi_{10}(0)>0$, then $u_{10}(t, \varphi)>0$ from $(2.1)_{10}$. Therefore, we have $u(t, \varphi) \in X_{0}$ for all $t>t_{0}$. This implies that, 
for any $\varphi \in \partial X_{0} \backslash\left(D_{1} \cup D_{2}\right)$, there exist some $n$, with $n \tau>t_{0}$, such that $P^{n}(\varphi) \notin \partial X_{0}$. Hence, $K_{\partial} \subset\left(D_{1} \cup D_{2}\right)$. This concludes the proof of Lemma D.2.

Lemma D.2 shows that $K_{\partial}=\left(D_{1} \cup D_{2}\right)$. Thus, from (D-2), it can verified that $\mathcal{P}^{n}(\phi), n \geq 0$, contains exactly two disease-free states, namely:

$$
\mathcal{T}_{0}=\left(S_{c}^{*}, 0,0,0,0,0,0,0, S_{a}^{*}, 0,0,0,0,0,0,0,0,0,0\right),
$$

and,

$$
\mathcal{E}_{0 N}=\left(S_{c}^{*}, 0,0,0,0,0,0,0, S_{a}^{*}, 0,0,0,0,0,0,0, M_{a}^{*}(t), M_{s}^{*}(t), 0\right),
$$

where $S_{c}^{*}, S_{a}^{*}, M_{a}^{*}(t)$ and $M_{s}^{*}(t)$ are as defined in Section 4 . Hence, the sets $A_{1}:=\left\{\mathcal{T}_{0}\right\}$ and $A_{2}:=\left\{\mathcal{E}_{0 N}\right\}$ are disjoint, compact and isolated invariant sets for the Poincaré map $\mathcal{P}$ in $K_{\partial}$, and $\bigcup_{\phi \in K_{\partial}} \omega(\phi)=\left\{A_{1}, A_{2}\right\}$ [36]. Furthermore, no subset of $\left\{A_{1}, A_{2}\right\}$ forms a cycle in $K_{\partial}$ (and, hence, in $\partial X_{0}$ ) [36]. In addition, it follows from the proof of Theorem 3.2 in [36] that, since $N_{v}^{*}(t)$ is a positive periodic solution with respect to $X_{0}$, then there exist a $\delta>0$ and an $\epsilon>0$ such that

$$
\limsup _{t \rightarrow \infty}\left|\Phi(n \tau) \phi-A_{1}\right| \geq \delta \text { and } \limsup _{t \rightarrow \infty}\left|\Phi(n \tau) \phi-A_{2}\right| \geq \epsilon \text { for all } \phi \in X_{0} .
$$

In view of the claims above, it follows that $A_{1}$ and $A_{2}$ are isolated invariant sets for $\mathcal{P}$ in $X$, and $W^{s}\left(A_{i}\right) \cap X_{0}=\emptyset$ for all $i=1,2$, where $W^{s}\left(A_{i}\right)$ is the stable set of $A_{i}$ for $\mathcal{P}$ [36]. In the case where $\mathbf{0}$ is not a solution of Equation (2.14), we can show that $K_{\partial}=D_{1}$ in a similar manner. It then follows that $\left\{A_{2}\right\}$ is the only compact invariant set for $\mathcal{P}$ in $K_{\partial}$ [36].

Hence, every trajectory in $K_{\partial}$ converges to either $A_{1}$ or $A_{2}$, and $\left\{A_{1}\right\}$ or $\left\{A_{2}\right\}$ are acyclic in $K_{\partial}$ [79]. It then follows from Theorem 1.3.1 in [79] (see also Theorem 2.2 in [78]) that $\mathcal{P}$ is uniformly-persistent with respect to $X_{0}$. Thus, it follows from Theorem 3.1.1 in [79], that the periodic semiflow $\Phi(t): X \rightarrow X$ is also uniformly-persistent to $X$ [36], where $\Phi(t) \varphi=u(t, \varphi)$. It then follows from Theorem 4.5 in [39] (see also Theorem 4.6 in [68] and Theorem 3.1 in [80]) that the model (2.1) admits a positive periodic solution denoted by $\mathcal{E}_{N}^{*}(t)=\Phi(t) \phi$ with $\phi \in X_{0}$.

It follows, from Theorem 4.5 in [39] (see also Theorem 2.1 in [78]), that $\mathcal{P}: X_{0} \rightarrow X_{0}$ has a compact global attractor, denoted by $\mathcal{A}_{0}$. Hence, $\mathcal{A}_{0}$ is invariant for $\mathcal{P}$ (that is, $\mathcal{A}_{0}=$ $\left.\mathcal{P}\left(\mathcal{A}_{0}\right)=\Phi(\tau) A_{0}\right)$. Furthermore, using the notation in [78], let $\mathcal{A}_{0}^{*}:=\bigcup_{t \in[0, \tau]} \Phi(t) \mathcal{A}_{0}$. Then, $\psi_{i}(0)>0$ for all $\psi \in \mathcal{A}_{0}^{*}, i \in[1,19]$ [36]. Since $\Phi(t) \phi \in X_{0}$, for all $t \geq 0$ and $\phi \in X_{0}$ (since $X_{0}$ is invariant), it follows that $\Phi(t) X_{0} \subset X_{0}$. Thus, $\mathcal{A}_{0}^{*} \subset X_{0}$ and $\limsup d\left(\Phi(t) \phi, \mathcal{A}_{0}^{*}\right)=0$ for all $\phi \in X_{0}[36,78]$. Also, it follows, by the continuity of $\Phi(t) \phi$ for $(t, \phi) \in[0, \infty) \times X_{0}$ and the compactness of $[0, \tau] \times \mathcal{A}_{0}[78]$, that $\mathcal{A}_{0}^{*}$ is compact in $X_{0}[36,78]$. Thus, $\inf _{\phi \in \mathcal{A}_{0}^{*}} d\left(\phi, \partial X_{0}\right)=$ $\min _{\phi \in \mathcal{A}_{0}^{*}} d\left(\phi, \partial X_{0}\right)>0[36,78]$. Consequently, there exists $q>0$ such that

$$
\begin{aligned}
& \liminf _{t \rightarrow \infty} \min \left(S_{c}(t, \phi), S_{c r}(t, \phi), E_{c}(t, \phi), E_{c r}(t, \phi), I_{c}(t, \phi), I_{c r}(t, \phi), R_{c}(t, \phi), R_{c r}(t, \phi), S_{a}(t, \phi),\right. \\
& \left.S_{a r}(t, \phi), E_{a}(t, \phi), E_{a r}(t, \phi), I_{a}(t, \phi), I_{a r}(t, \phi), R_{a}(t, \phi), R_{a r}(t, \phi), M_{a}(t, \phi), M_{s}(t, \phi), M_{i}(t, \phi)\right) \\
& =\liminf _{t \rightarrow \infty} d\left(\phi, \partial X_{0}\right) \geq q, \text { for all } \phi \in X_{0} .
\end{aligned}
$$

In particular, $\liminf _{t \rightarrow \infty} \min \left(\Phi(t) \phi^{*}\right) \geq q$, and hence, $u_{i}(t, \phi)>0,1 \leq i \leq 19$ for all $t \geq 0$. 


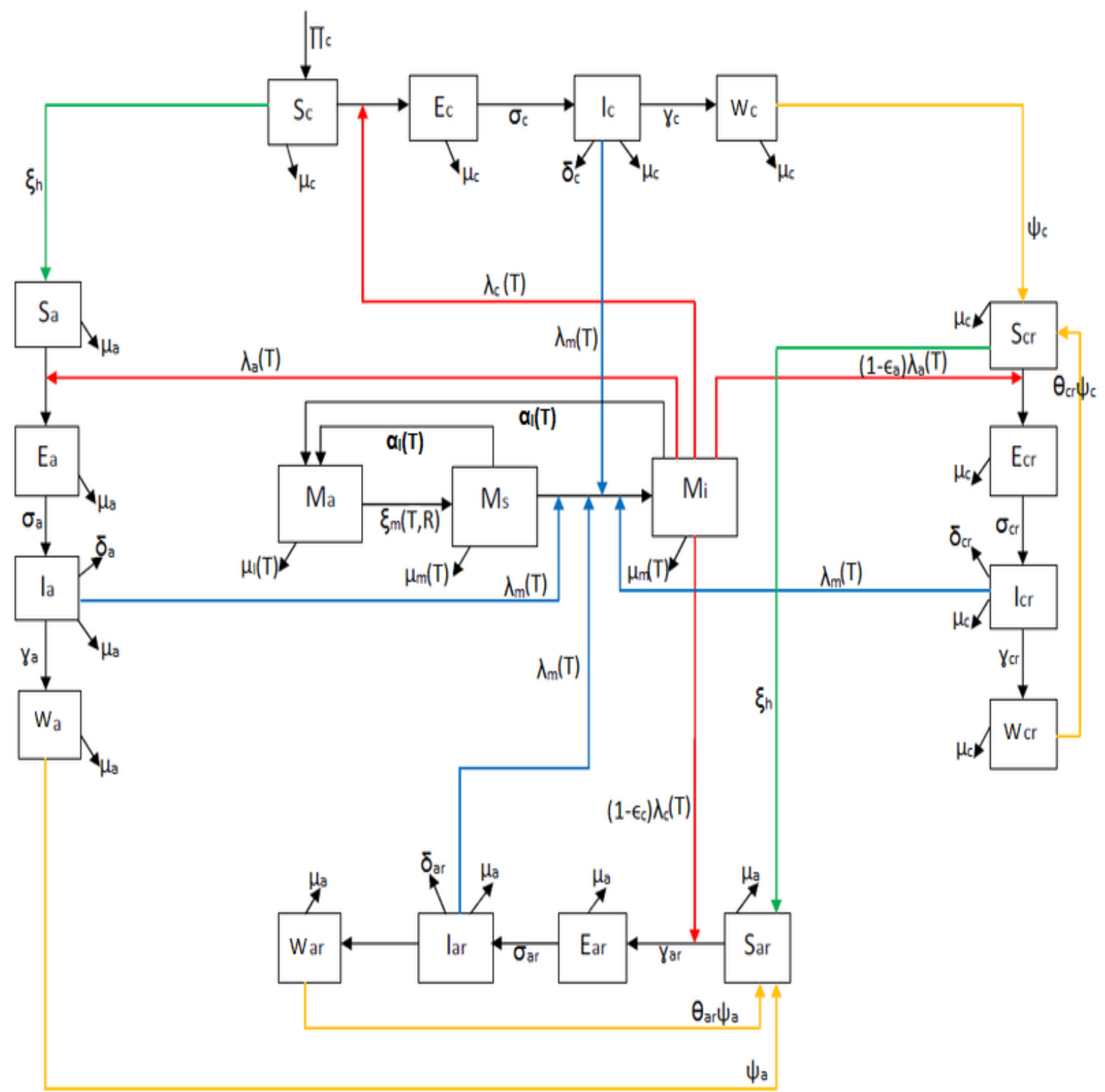

Figure 1: Flow diagram of the non-autonomous model (2.1). 

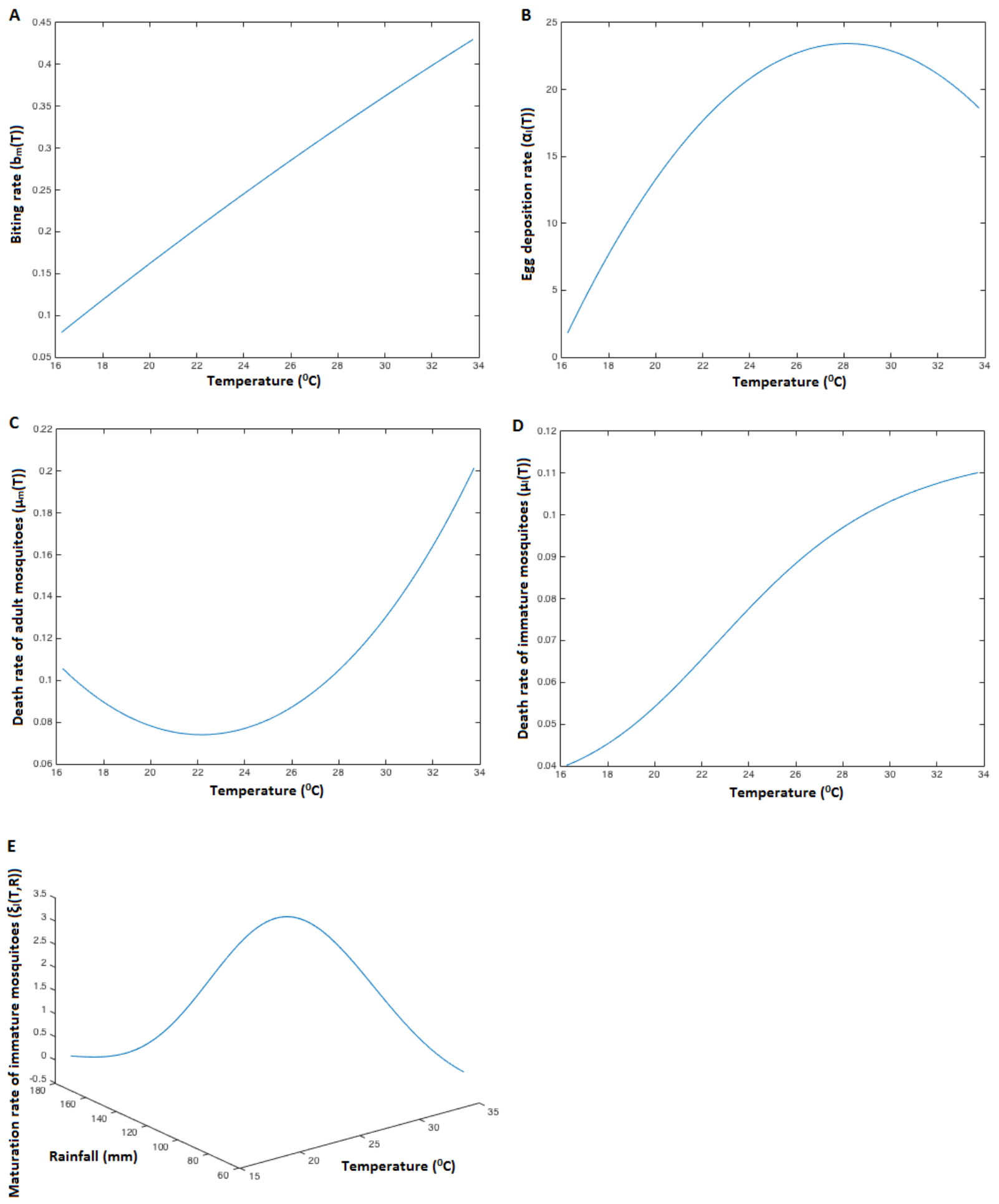

Figure 2: Profile of temperature- and rainfall-dependent parameters of the model (2.1). (A) Biting rate of mosquitoes, $b_{m}(T)(\mathrm{B})$ Egg deposition rate, $\mu_{b}(T)(\mathrm{C})$ Death rate of adult mosquitoes, $\mu_{m}(T)$ (D) Death rate of immature mosquitoes, $\mu_{l}(T)$ (E) Maturation rate of immature mosquitoes, $\xi_{l}(T, R)$. 


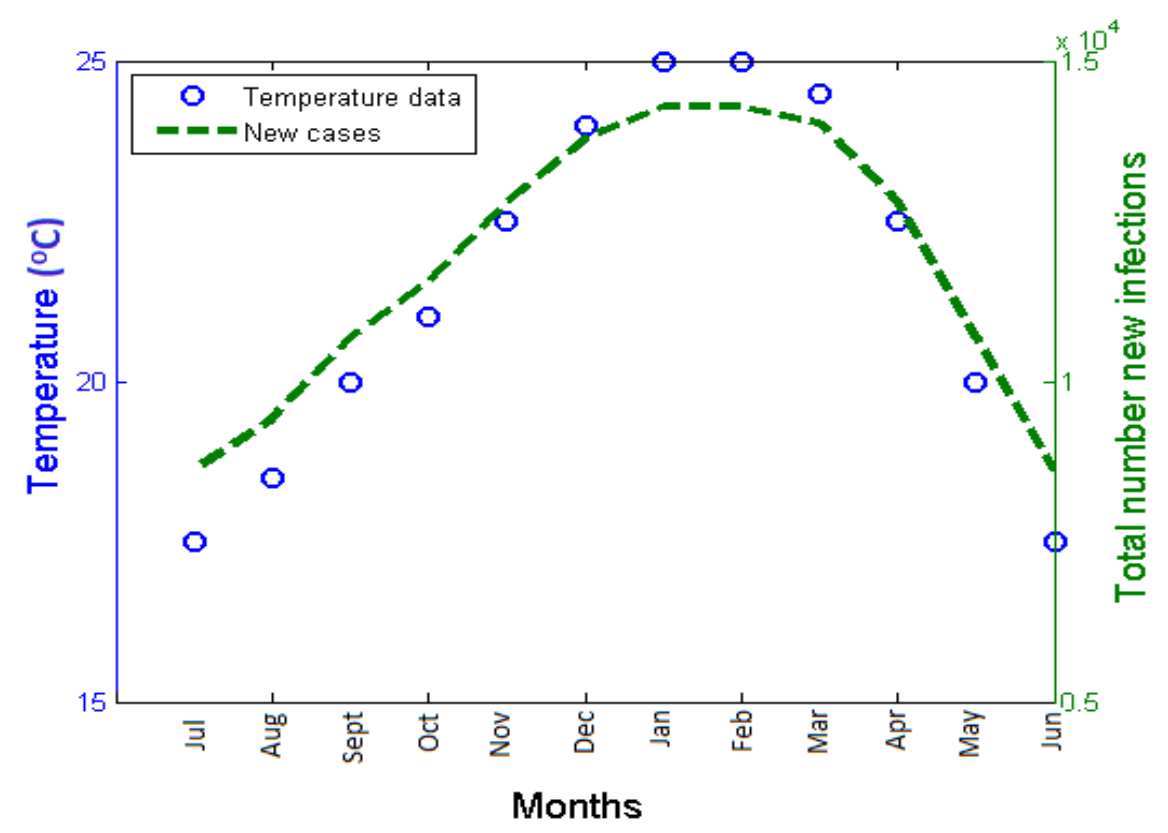

Figure 3: Effect of temperature on disease dynamics (for fixed mean monthly rainfall $R(t)=$ $86.63 \mathrm{~mm}$ ). Simulation of the model (2.1) showing the total number of new infections as a function of time for various temperature values in the range $[17.5,25.1]^{\circ} \mathrm{C}$. Parameter values used in the simulations are as in Tables 2, 5 and 6.

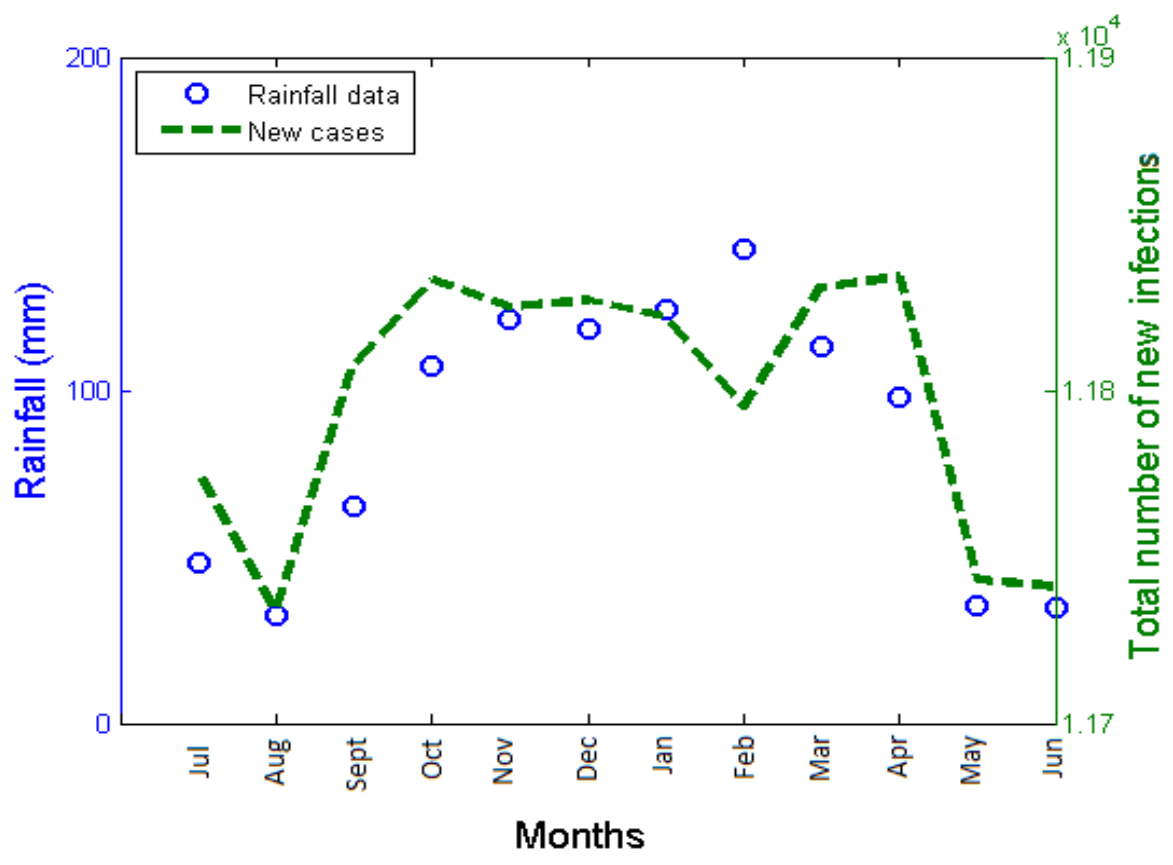

Figure 4: Effect of rainfall on disease dynamics (for fixed mean monthly temperature $21.5^{\circ} \mathrm{C}$ ). Simulation of the model (2.1) showing the total number of new infections as a function of time for various levels of rainfall in the range $[32.3,142.2] \mathrm{mm}$. Parameter values used in the simulations are as in Tables 2, 5 and 6. 


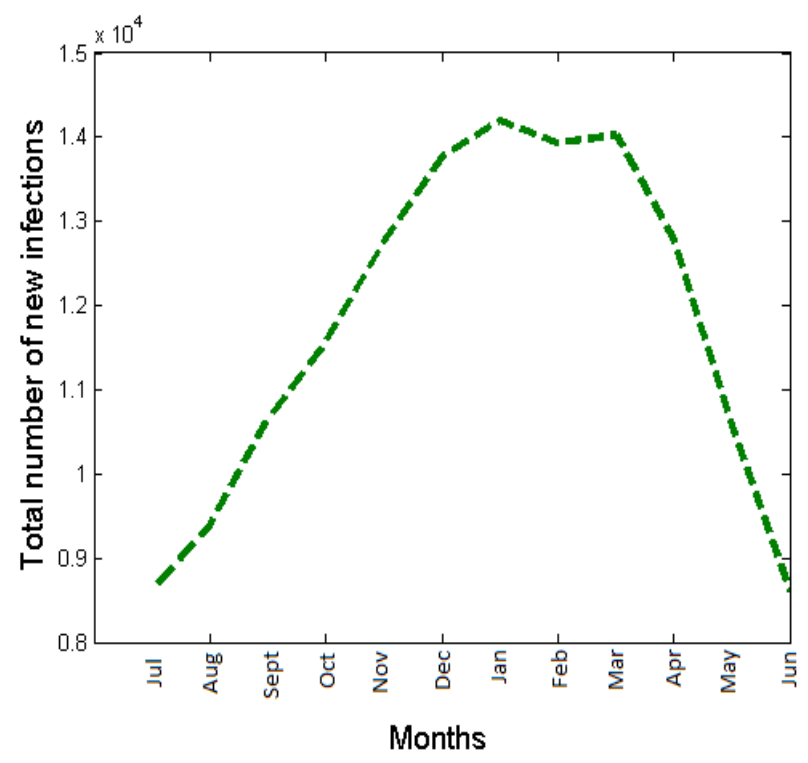

Figure 5: Simulation of the non-autonomous model (2.1) showing the total number of new infections for various values of temperature and rainfall values in the range $T \in[17.5,25.0]^{\circ} \mathrm{C}$ and $R \in[32.3,142.2] \mathrm{mm}$. All parameters are as given in Tables 2,5 , and 6 .

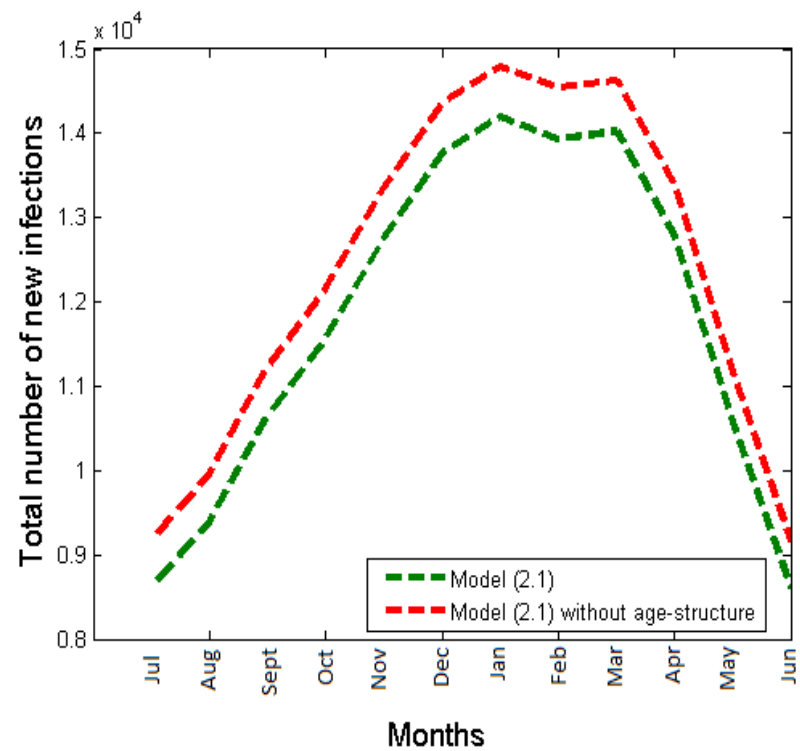

(a)

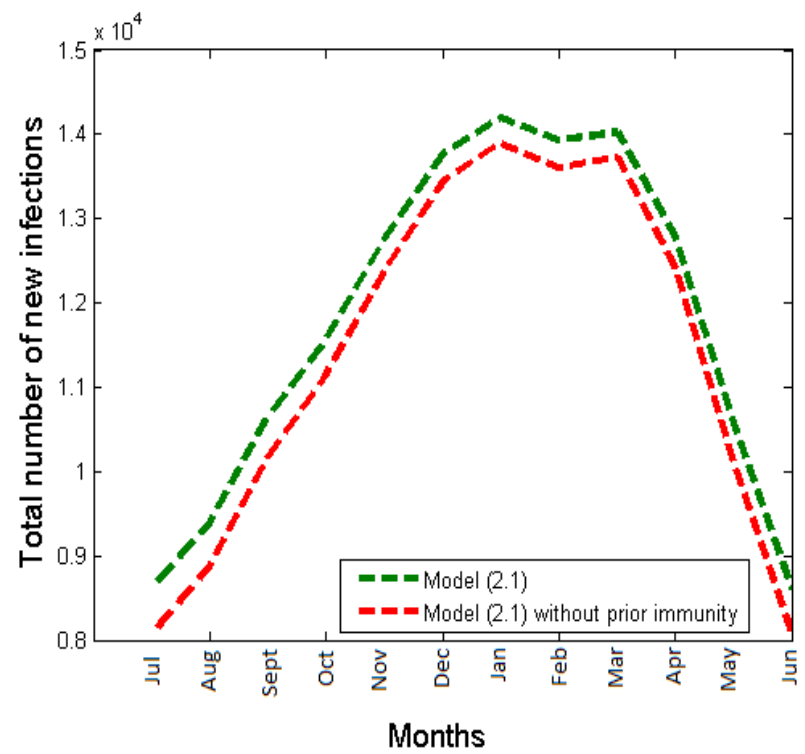

(b)

Figure 6: Simulation of the non-autonomous model (2.1) showing the total number of new infections for various values of temperature and rainfall values in the range $T \in[17.5,25.0]^{\circ} \mathrm{C}$ and $R \in[32.3,142.2] \mathrm{mm}$ and the: (a) marginal effect of age-structure; (b) marginal effect of classes of reduced susceptibility due to prior infection to models for malaria transmission dynamics. All parameters are as given in Tables 2, 5, and 6 . 


\begin{tabular}{|c|c|}
\hline Variables & Description \\
\hline$S_{c}(t)$ & Population of susceptible children \\
\hline$S_{c r}(t)$ & Population of susceptible children with immunity against re-infection \\
\hline$E_{c}(t)$ & Population of latently-infected children \\
\hline$E_{c r}(t)$ & Population of latently-infected children with immunity against re-infection \\
\hline$I_{c}(t)$ & Population of infectious children \\
\hline$I_{c r}(t)$ & Population of infectious children with immunity against re-infection \\
\hline$W_{c}(t)$ & Population of treated children \\
\hline$W_{c r}(t)$ & Population of recovered children with immunity against re-infection \\
\hline$S_{a}(t)$ & Population of susceptible adults \\
\hline$S_{a r}(t)$ & Population of susceptible adults with immunity against re-infection \\
\hline$E_{a}(t)$ & Population of latently-infected adults \\
\hline$E_{a r}(t)$ & Population of latently-infected adults with immunity against re-infection \\
\hline$I_{a}(t)$ & Population of infectious adults \\
\hline$I_{a r}(t)$ & Population of infectious adults with immunity against re-infection \\
\hline$W_{a}(t)$ & Population of recovered adults \\
\hline$W_{a r}(t)$ & Population of recovered adults with immunity against re-infection \\
\hline$M_{a}(t)$ & Population of aquatic mosquitoes \\
\hline$M_{s}(t)$ & Population of susceptible mosquitoes \\
\hline$M_{i}(t)$ & Population of infectious mosquitoes \\
\hline$\lambda_{j}(T),(j=a, c, m)$ & $\begin{array}{l}\text { Infection rate for susceptible children, susceptible adults } \\
\text { and susceptible mosquitoes }\end{array}$ \\
\hline Parameters & Description \\
\hline$\Pi_{c}$ & Birth rate of children \\
\hline$\mu_{h}$ & Per capita death rate for humans \\
\hline$\alpha_{l}(T)$ & Per capita egg deposition rate \\
\hline$\mu_{l}(T), \mu_{m}(T)$ & Per capita death rate for aquatic and adult mosquitoes \\
\hline$\sigma_{i}(i=c, a, c r, a r)$ & Progression rate from exposed to symptomatic class \\
\hline$\gamma_{i}$ & $\begin{array}{l}\text { Recovery rate of infectious humans without }(i=c, a) \text { and with }(i=c r, a r) \\
\text { prior immunity to malaria }\end{array}$ \\
\hline$\delta_{i}$ & Disease-induced death rate of infectious humans \\
\hline$\in_{c}, \in_{a}$ & Modification parameter for reduction in infectivity of malaria \\
\hline$\xi_{h}$ & Maturation rate of children to adult \\
\hline$\xi_{l}(T, R)$ & Maturation rate of immature mosquitoes \\
\hline$\psi_{c}, \psi_{a}$ & Transmission rate of recovered individuals into partially immuned classes \\
\hline$\theta_{c r}, \theta_{a r}$ & Modification parameter for increase in rate of infection acquired immunity \\
\hline$\eta$ & Modification parameter for reduction in infectiousness \\
\hline$b_{c}(T)$ & Per capita biting rate of mosquitoes on susceptible children \\
\hline$b_{a}(T)$ & Per capita biting rate of mosquitoes on susceptible adults \\
\hline$b_{m}(T)$ & Per capita biting rate of susceptible mosquitoes on infectious humans \\
\hline$\beta_{c}$ & Probability of malaria transmission from infected mosquitoes to susceptible childrer \\
\hline$\beta_{a}$ & Probability of malaria transmission from infected mosquitoes to susceptible adults \\
\hline$\beta_{m}$ & Probability of infection from infected humans to susceptible mosquitoes \\
\hline$K_{v}$ & Vector carrying capacity \\
\hline$p_{M E}, p_{M L}, p_{M P}$ & Maximum daily survival probability of egg, larva and pupa \\
\hline$B(T)$ & The lifetime number of eggs laid \\
\hline$\tau_{E A}(T)$ & The development time from egg to adult mosquito \\
\hline$E F D(T)$ & The number of eggs laid per female per day \\
\hline$R_{L}$ & Rainfall threshold \\
\hline
\end{tabular}

Table 1: Description of variables and parameters of the model (2.1). 


\begin{tabular}{llll}
\hline \hline Parameters & Baseline Value & Range & Reference \\
\hline \hline$\Pi_{c}$ & $540 /$ day & $(510-570) /$ day & Adapted from $[64,70]$ \\
$\mu_{h}$ & $0.00005 /$ day & $(0.00003-0.00006) /$ day & {$[70]$} \\
$\alpha_{l}$ & $1.84 /$ day & $(1-500) /$ day & {$[1,35,36,60]$} \\
$\mu_{l}$ & $0.1041 /$ day & $0.071-0.25 /$ day & {$[1,35,36,60]$} \\
$\mu_{m}$ & $0.05 /$ day & $0.04762-0.07143 /$ day & {$[1,12,35,48,60]$} \\
$\sigma_{c}, \sigma_{a}$ & $0.083 /$ day & $(0.06-0.203) /$ day & {$[1,12,48]$} \\
$\sigma_{c r}$ & $0.055 /$ day & $(0.050-0.20) /$ day & \\
$\sigma_{a r}$ & $0.051 /$ day & $(0.050-0.20) /$ day & \\
$\gamma_{c}$ & $0.0025 /$ day & $(0.0014-0.017) /$ day & \\
$\gamma_{a}$ & $0.0028 /$ day & $(0.001-0.023) /$ day & {$[1,12,48]$} \\
$\gamma_{c r}$ & $0.0080 /$ day & $(0.0012-0.02) /$ day & \\
$\gamma_{a r}$ & $0.0090 /$ day & $(0.001-0.03) /$ day & \\
$\delta_{i}(i=a, a r)$ & $0.0002 /$ day & $(0.00001-0.0004) /$ day & {$[1,12,48]$} \\
$\delta_{j}(j=c, c r)$ & $0.0003 /$ day & $(0.00001-0.0005) /$ day & \\
$\epsilon_{c}, \epsilon_{a}$ & 0.4 & $(0.3-0.5)$ & \\
$\xi_{h}$ & $0.000161 /$ day & $(0.000144-0.000183) /$ day & \\
$\xi_{l}$ & $0.343 /$ day & $(0.333-1) /$ day & \\
$\psi_{c}, \psi_{a}$ & 0.002 & $(0.0011-0.005) /$ day & \\
$\theta_{c r}, \theta_{a r}$ & 0.004 & $(0.003-0.005)$ & \\
$\eta$ & 0.4 & $(0.3-0.5)$ & {$[53]$} \\
$b_{m}$ & $0.29 /$ day & $(0.10-1.0) /$ day & {$[1,12,48]$} \\
$\beta_{c}, \beta_{a}$ & $0.24 /$ day & $(0.072-0.64) /$ day & {$[1,12,48]$} \\
$\beta_{m}$ & $0.022 /$ day & $(0.027-0.64) /$ day & {$[1,12]$} \\
$K_{v}$ & 40000 & $\left(50-3 \times 10^{5}\right)$ & {$[1,35,60]$} \\
$R_{L}$ & 200 mm & & \\
$p_{M E}$ & 0.9 & & \\
$p_{M L}$ & 0.25 & & \\
$p_{M P}$ & 0.75 & & \\
\hline \hline & & & \\
\hline
\end{tabular}

Table 2: Values and ranges of the parameters of the autonomous version of the model (2.1). 


\begin{tabular}{|c|c|c|c|c|c|c|}
\hline Parameters & $\begin{array}{l}I_{c} \text { Class } \\
\end{array}$ & $I_{c r}$ Class & $I_{a}$ Class & $I_{a r}$ Class & $M_{i}$ Class & $\overline{\mathcal{R}_{0}}$ \\
\hline$\overline{\Pi_{c}}$ & -0.0561 & +0.0550 & +0.1157 & -0.0144 & -0.0184 & -0.08 \\
\hline$b_{m}$ & +0.6393 & +0.4189 & +0.6451 & +0.3601 & +0.4030 & +0.86 \\
\hline$\beta_{c}$ & +0.3793 & +0.0351 & +0.1573 & +0.1761 & -0.1418 & +0.18 \\
\hline$\beta_{a}$ & +0.0731 & +0.0516 & +0.0265 & +0.2795 & -0.0664 & +0.47 \\
\hline$\beta_{m}$ & +0.4903 & +0.2679 & +0.4210 & +0.2009 & +0.3083 & +0.69 \\
\hline$\epsilon_{c}$ & -0.0297 & -0.0510 & -0.0297 & -0.0004 & -0.0024 & - \\
\hline$\epsilon_{a}$ & -0.0571 & -0.0391 & -0.0571 & -0.0310 & +0.0325 & - \\
\hline$\mu_{h}$ & +0.1557 & +0.1369 & +0.1605 & -0.1048 & -0.0241 & +0.25 \\
\hline$\alpha_{l}$ & +0.0161 & +0.0324 & +0.0077 & +0.0016 & +0.0264 & -0.022 \\
\hline$\mu_{l}$ & -0.0247 & +0.0595 & -0.0440 & -0.0148 & +0.1029 & -0.032 \\
\hline$\mu_{m}$ & -0.2280 & -0.0017 & -0.2312 & -0.0471 & -0.0221 & -0.31 \\
\hline$\xi_{h}$ & +0.0050 & +0.0032 & +0.0646 & -0.0700 & +0.0161 & +0.024 \\
\hline$\xi_{l}$ & +0.2273 & +0.1615 & +0.2093 & +0.1983 & +0.0458 & +0.41 \\
\hline$\sigma_{c}$ & -0.0059 & +0.0446 & -0.0916 & -0.0252 & -0.0431 & +0.0022 \\
\hline$\sigma_{a}$ & +0.0572 & +0.0041 & +0.1143 & -0.0258 & -0.0495 & +0.0053 \\
\hline$\sigma_{c r}$ & -0.0098 & +0.0271 & -0.0264 & -0.0507 & +0.0126 & - \\
\hline$\sigma_{a r}$ & -0.0198 & -0.0402 & -0.1224 & -0.0171 & +0.0069 & - \\
\hline$\delta_{c}$ & -0.0193 & +0.0373 & -0.0193 & +0.0721 & -0.0243 & +0.0021 \\
\hline$\delta_{a}$ & +0.0515 & -0.0180 & -0.0072 & -0.0255 & +0.0059 & +0.072 \\
\hline$\delta_{c r}$ & +0.0782 & -0.0454 & -0.0782 & -0.0195 & +0.0485 & - \\
\hline$\delta_{a r}$ & +0.0001 & +0.0375 & +0.0267 & +0.0017 & +0.0060 & - \\
\hline$\psi_{c}$ & -0.1349 & -0.0372 & -0.1592 & +0.0494 & +0.0756 & - \\
\hline$\psi_{a}$ & +0.0159 & -0.0870 & +0.0159 & +0.0559 & +0.0315 & - \\
\hline$\theta_{c r}$ & +0.0702 & -0.0288 & +0.0205 & +0.0335 & -0.0081 & - \\
\hline$\theta_{a r}$ & +0.00604 & -0.0350 & -0.0173 & +0.0165 & +0.0025 & - \\
\hline$\gamma_{c}$ & -0.4761 & -0.7429 & -0.4207 & -0.0621 & -0.0344 & -0.19 \\
\hline$\gamma_{a}$ & -0.4690 & -0.1232 & -0.5164 & -0.8003 & -0.0303 & -0.56 \\
\hline$\gamma_{c r}$ & -0.2203 & -0.0332 & -0.2197 & +0.0427 & -0.0496 & - \\
\hline$\gamma_{a r}$ & -0.2032 & -0.0127 & -0.2201 & -0.0947 & -0.0146 & - \\
\hline$K_{v}$ & +0.8909 & +0.7014 & +0.9063 & +0.6251 & +0.6314 & +0.78 \\
\hline$\eta$ & -0.0306 & +0.0242 & -0.04057 & -0.0887 & -0.0925 & - \\
\hline
\end{tabular}

Table 3: PRCC values for the parameters of the autonomous version of the model (2.1) using total number of infectious children, adults, mosquitoes and $\mathcal{R}_{0}$ as output. The top (most dominant) parameters that affect the dynamics of the model with respect to each of the six response function are highlighted in bold font.

Notation: a line (-) indicates the parameter is not in the expression for $\mathcal{R}_{0}$. 


\begin{tabular}{|c|c|c|c|c|c|c|c|c|c|c|c|c|}
\hline \hline Month & Jul & Aug & Sept & Oct & Nov & Dec & Jan & Feb & Mar & Apr & May & Jun \\
\hline Temperature $\left({ }^{\circ} \mathrm{C}\right)$ & 17.5 & 18.5 & 20.0 & 21.0 & 22.5 & 24.0 & 25.0 & 25.0 & 24.5 & 22.5 & 20.0 & 17.5 \\
\hline Rainfall $(\mathrm{mm})$ & 48.2 & 32.3 & 65.2 & 107.1 & 121.1 & 118.3 & 124 & 142.2 & 113 & 98.1 & 35.4 & 34.7 \\
\hline \hline
\end{tabular}

Table 4: Monthly mean temperature (in ${ }^{\circ} \mathrm{C}$ ) and rainfall (in $\mathrm{mm}$ ) for KwaZulu-Natal Province of South Africa [18].

\begin{tabular}{|c|c|c|c|c|c|c|c|c|c|}
\hline \hline Temperature $\left({ }^{\circ} \mathrm{C}\right)$ & $b_{m}(T)$ & $*$ & $\mu_{m}(T)$ & $*$ & $\alpha_{l}(T)$ & $*$ & $\mu_{l}(T)$ & $*$ & $\xi_{l}(T)_{86.63 m m}$ \\
\hline $16-20$ & $0.074-0.162$ & 0.12 & $0.078-0.108$ & 0.10 & $0.892-13.3$ & 14.2 & $0.039-0.054$ & 0.05 & $0.024-1.3541$ \\
$20-24$ & $0.162-0.245$ & 0.20 & $0.074-0.078$ & 0.07 & $13.3-20.81$ & 17.1 & $0.054-0.078$ & 0.07 & $1.3541-3.340$ \\
$24-28$ & $0.245-0.324$ & 0.28 & $0.077-0.105$ & 0.09 & $20.81-23.43$ & 22.1 & $0.078-0.097$ & 0.09 & $2.7167-3.4367$ \\
$28-32$ & $0.324-0.399$ & 0.36 & $0.105-0.164$ & 0.13 & $21.15-23.43$ & 22.3 & $0.097-0.108$ & 0.10 & $0.9854-2.7167$ \\
$32-36$ & $0.399-0.469$ & 0.43 & $0.164-0.261$ & 0.21 & $13.97-21.15$ & 17.6 & $0.108-0.112$ & 0.11 & $0.0091-0.9854$ \\
\hline \hline
\end{tabular}

Table 5: Ranges of temperature-dependent parameters of the model (2.1) for fixed temperature values in the range $[16,36]^{\circ} \mathrm{C}$ (with corresponding baseline values) and fixed mean rainfall value $(R(t)=86.63 \mathrm{~mm})$.

*- Baseline value.

\begin{tabular}{|c|c|c|c|c|c|c|c|}
\hline \hline Temperature & Rainfall & $\xi_{l}(T, R)$ & $*$ & Temperature & Rainfall & $\xi_{l}(T, R)$ & $*$ \\
\hline \multirow{3}{*}{$16-20^{\circ} \mathrm{C}$} & $30-60 \mathrm{~mm}$ & $0.0003-0.8472$ & 0.42 & & $30-60 \mathrm{~mm}$ & $0.1869-2.0897$ & 1.14 \\
& $60-90 \mathrm{~mm}$ & $0.015-1.3869$ & 0.70 & $20-24^{\circ} \mathrm{C}$ & $60-90 \mathrm{~mm}$ & $0.8472-3.4210$ & 2.13 \\
& $90-120 \mathrm{~mm}$ & $0.022-1.4294$ & 0.73 & & $90-120 \mathrm{~mm}$ & $1.2646-3.5257$ & 2.40 \\
& $120-150 \mathrm{~mm}$ & $0.011-1.2646$ & 0.64 & & $120-150 \mathrm{~mm}$ & $0.6030-3.1193$ & 1.86 \\
\hline \hline \multirow{3}{*}{$24-28^{\circ} \mathrm{C}$} & $30-60 \mathrm{~mm}$ & $0.4677-2.0897$ & 1.28 & & $30-60 \mathrm{~mm}$ & $0.1380-1.6997$ & 0.92 \\
& $60-90 \mathrm{~mm}$ & $1.5658-3.4210$ & 2.49 & $28-32^{\circ} \mathrm{C}$ & $60-90 \mathrm{~mm}$ & $1.0093-2.7825$ & 1.90 \\
& $90-120 \mathrm{~mm}$ & $2.5372-3.6264$ & 3.08 & & $90-120 \mathrm{~mm}$ & $0.9203-2.8677$ & 1.89 \\
& $120-150 \mathrm{~mm}$ & $1.2098-3.2053$ & 2.21 & & $120-150 \mathrm{~mm}$ & $0.4389-2.5372$ & 1.49 \\
\hline \hline
\end{tabular}

Table 6: Ranges of temperature- and rainfall-dependent parameter of the model (2.1) for fixed temperature values in the range $[16,32]^{\circ} \mathrm{C}$ and rainfall in the range $[30,150] \mathrm{mm}$ (with $\left.R_{L E}=200 \mathrm{~mm}\right)$.

$*-$ Baseline value. 


\begin{tabular}{|c|c|c|c|c|c|c|c|c|}
\hline \multirow{2}{*}{ Parameters } & \multicolumn{2}{|c|}{$16-20^{\circ} \mathrm{C}$} & \multicolumn{2}{c|}{$20-24^{\circ} \mathrm{C}$} & \multicolumn{2}{c|}{$24-28^{\circ} \mathrm{C}$} & \multicolumn{2}{c|}{$28-32^{\circ} \mathrm{C}$} \\
& $60-90 \mathrm{~mm}$ & $90-120 \mathrm{~mm}$ & $60-90 \mathrm{~mm}$ & $90-120 \mathrm{~mm}$ & $60-90 \mathrm{~mm}$ & $90-120 \mathrm{~mm}$ & $60-90 \mathrm{~mm}$ & $90-120 \mathrm{~mm}$ \\
\hline$\Pi_{c}$ & -0.045 & -0.037 & -0.054 & -0.06 & -0.089 & -0.038 & -0.033 & -0.10 \\
\hline$b_{m}$ & $+\mathbf{0 . 5 5}$ & $\mathbf{+ 0 . 5 7}$ & +0.35 & +0.40 & +0.28 & +0.27 & +0.19 & +0.23 \\
\hline$\beta_{c}$ & +0.18 & +0.22 & +0.20 & +0.24 & +0.26 & +0.17 & +0.24 & +0.19 \\
\hline$\beta_{a}$ & +0.47 & +0.53 & $\mathbf{+ 0 . 5 9}$ & $\mathbf{+ 0 . 5 6}$ & $+\mathbf{0 . 5 7}$ & $+\mathbf{0 . 5 8}$ & $+\mathbf{0 . 5 6}$ & $+\mathbf{0 . 5 7}$ \\
\hline$\beta_{m}$ & $\mathbf{+ 0 . 7 2}$ & $\mathbf{+ 0 . 7 4}$ & $\mathbf{+ 0 . 7 8}$ & $\mathbf{+ 0 . 7 4}$ & $+\mathbf{0 . 7 9}$ & $+\mathbf{0 . 7 9}$ & $+\mathbf{0 . 7 8}$ & $+\mathbf{0 . 7 8}$ \\
\hline$\mu_{h}$ & +0.28 & +0.26 & +0.34 & +0.34 & +0.33 & +0.30 & +0.24 & +0.28 \\
\hline$\alpha_{l}$ & +0.041 & +0.04 & +0.022 & +0.043 & +0.015 & -0.006 & -0.019 & +0.015 \\
\hline$\mu_{l}$ & -0.0057 & +0.029 & +0.044 & +0.013 & -0.031 & -0.015 & +0.052 & -0.032 \\
\hline$\mu_{m}$ & -0.27 & -0.28 & -0.042 & -0.031 & -0.35 & -0.3 & -0.42 & -0.41 \\
\hline$\xi_{h}$ & +0.013 & -0.0023 & +0.035 & -0.065 & +0.0072 & +0.034 & -0.01 & -0.04 \\
\hline$\xi_{l}$ & $+\mathbf{0 . 7 6}$ & $\mathbf{+ 0 . 7 7}$ & $+\mathbf{0 . 5 7}$ & $+\mathbf{0 . 4 2}$ & $+\mathbf{0 . 4 2}$ & $+\mathbf{0 . 4 0}$ & $+\mathbf{0 . 4 5}$ & $+\mathbf{0 . 5 0}$ \\
\hline$\sigma_{c}$ & +0.029 & +0.015 & -0.04 & -0.063 & -0.022 & -0.012 & +0.015 & +0.017 \\
\hline$\sigma_{a}$ & -0.022 & -0.0055 & +0.017 & -0.0032 & -0.036 & +0.057 & -0.042 & -0.0011 \\
\hline$\delta_{c}$ & +0.006 & -0.0074 & -0.035 & -0.05 & -0.052 & -0.067 & -0.014 & -0.017 \\
\hline$\delta_{a}$ & +0.034 & -0.053 & -0.058 & +0.016 & -0.026 & -0.022 & -0.031 & -0.085 \\
\hline$\gamma_{c}$ & -0.29 & -0.33 & -0.30 & -0.32 & -0.34 & -0.34 & -0.35 & -0.29 \\
\hline$\gamma_{a}$ & $\mathbf{0 . 5 9}$ & $\mathbf{- 0 . 5 7}$ & $\mathbf{- 0 . 6 6}$ & $\mathbf{- 0 . 6 4}$ & $\mathbf{- 0 . 6 7}$ & $\mathbf{- 0 . 6 7}$ & $\mathbf{- 0 . 6 3}$ & $\mathbf{- 0 . 6 5}$ \\
\hline$K_{v}$ & $+\mathbf{0 . 7 6}$ & $\mathbf{+ 0 . 7 9}$ & $+\mathbf{0 . 8 3}$ & $+\mathbf{0 . 8 2}$ & $+\mathbf{0 . 8 4}$ & $+\mathbf{0 . 8 2}$ & $+\mathbf{0 . 8 2}$ & $+\mathbf{0 . 8 3}$ \\
\hline
\end{tabular}

Table 7: PRCC values for the temperature- and rainfall dependent parameters of the model (2.1), using $\mathcal{R}_{0}$ as output with temperature values $T \in[16-20]^{\circ} \mathrm{C}$ and rainfall $R \in[60-120]$ $\mathrm{mm}$. The top (most dominant) parameters that affect the dynamics of the model with respect to each of the four temperature ranges chosen are highlighted in bold font. 


\begin{tabular}{|c|c|c|c|c|c|c|c|c|}
\hline \multirow{2}{*}{ Parameters } & \multicolumn{2}{|c|}{$16-20^{\circ} \mathrm{C}$} & \multicolumn{2}{|c|}{$20-24^{\circ} \mathrm{C}$} & \multicolumn{2}{|c|}{$24-28^{\circ} \mathrm{C}$} & \multicolumn{2}{|c|}{$28-32^{\circ} \mathrm{C}$} \\
\hline & $60-90 \mathrm{~mm}$ & $90-120 \mathrm{~mm}$ & $60-90 \mathrm{~mm}$ & $90-120 \mathrm{~mm}$ & $60-90 \mathrm{~mm}$ & $90-120 \mathrm{~mm}$ & $60-90 \mathrm{~mm}$ & $90-120 \mathrm{~mm}$ \\
\hline$\Pi_{c}$ & +0.0659 & -0.0174 & +0.0057 & -0.0123 & -0.0932 & -0.0421 & -0.0098 & -0.0122 \\
\hline$b_{m}$ & +0.2993 & +0.2345 & +0.1474 & +0.1563 & +0.1105 & +0.1605 & +0.1488 & +0.0325 \\
\hline$\beta_{c}$ & +0.2147 & +0.2619 & +0.3216 & +0.3549 & +0.3462 & +0.3320 & +0.3763 & +0.3673 \\
\hline$\beta_{a}$ & +0.1222 & +0.1053 & +0.1051 & +0.0719 & +0.0496 & +0.0329 & +0.0462 & +0.083 \\
\hline$\beta_{m}$ & +0.4453 & +0.4346 & +0.4292 & +0.4383 & +0.4656 & +0.4694 & +0.4527 & +0.4531 \\
\hline$\epsilon_{c}$ & -0.0567 & -0.1050 & -0.1002 & -0.1045 & -0.0013 & -0.0454 & +0.0324 & -0.0761 \\
\hline$\epsilon_{a}$ & -0.0183 & +0.0243 & +0.0517 & -0.0262 & -0.0071 & +0.0253 & +0.0284 & -0.0117 \\
\hline$\mu_{h}$ & +0.1904 & +0.1802 & +0.2323 & +0.2408 & +0.2608 & +0.2789 & +0.1698 & +0.2007 \\
\hline$\alpha_{l}$ & +0.0217 & +0.0406 & +0.0253 & +0.0577 & +0.0175 & -0.0144 & +0.0303 & +0.0134 \\
\hline$\mu_{l}$ & +0.0720 & -0.0249 & +0.02663 & -0.0076 & +0.0042 & -0.0195 & +0.1307 & -0.0287 \\
\hline$\mu_{m}$ & -0.0987 & -0.1712 & -0.0057 & -0.069 & -0.1028 & -0.2036 & -0.2011 & -0.2519 \\
\hline$\xi_{h}$ & +0.0619 & -0.0276 & +0.035 & -0.0394 & +0.0862 & -0.0127 & +0.0577 & -0.0051 \\
\hline$\xi_{l}$ & +0.4415 & +0.4314 & +0.2581 & +0.2435 & +0.1920 & +0.0717 & +0.1845 & +0.1598 \\
\hline$\sigma_{c}$ & -0.01348 & +0.0544 & -0.0156 & +0.0692 & -0.0114 & +0.0217 & -0.0067 & +0.0505 \\
\hline$\sigma_{a}$ & +0.0233 & -0.0191 & +0.0116 & -0.0570 & -0.0039 & +0.0614 & +0.0274 & -0.0167 \\
\hline$\sigma_{c r}$ & -0.1174 & -0.1125 & -0.1837 & -0.1250 & -0.1615 & -0.1494 & -0.1773 & -0.1261 \\
\hline$\sigma_{a r}$ & +0.0537 & +0.0591 & +0.0328 & +0.0682 & -0.0327 & -0.0461 & -0.0702 & +0.0419 \\
\hline$\delta_{c}$ & -0.0473 & -0.0252 & +0.0450 & -0.05701 & -0.0083 & -0.0249 & -0.0341 & -0.0844 \\
\hline$\delta_{a}$ & +0.0304 & -0.0502 & -0.0339 & -0.00069 & -0.0035 & +0.0255 & +0.0126 & +0.0260 \\
\hline$\delta_{c r}$ & -0.0333 & -0.00895 & +0.0324 & +0.00288 & -0.0248 & -0.0393 & +0.0318 & -0.0038 \\
\hline$\delta_{a r}$ & +0.031 & +0.0315 & -0.1353 & +0.0746 & -0.0313 & -0.0452 & +0.0112 & -0.0847 \\
\hline$\psi_{c}$ & -0.2068 & -0.2034 & -0.2014 & -0.1566 & -0.137 & -0.1341 & -0.0724 & -0.1369 \\
\hline$\psi_{a}$ & -0.02501 & +0.0082 & +0.0314 & -0.0035 & -0.0601 & -0.0350 & -0.0285 & -0.0466 \\
\hline$\theta_{c r}$ & +0.0109 & +0.0182 & +0.0182 & +0.0149 & +0.0198 & +0.0025 & +0.0443 & -0.0248 \\
\hline$\theta_{a r}$ & -0.0282 & +0.0102 & +0.0102 & -0.0132 & -0.0543 & +0.0179 & +0.0412 & +0.0382 \\
\hline$\gamma_{c}$ & -0.6337 & -0.6054 & -0.5068 & -0.5232 & -0.5396 & -0.5325 & -0.5479 & -0.5352 \\
\hline$\gamma_{a}$ & -0.5734 & -0.5940 & -0.5290 & -0.4888 & -0.5229 & -0.5615 & -0.5811 & -0.5563 \\
\hline$\gamma_{c r}$ & -0.2383 & -0.2217 & -0.2515 & -0.2766 & -0.2597 & -0.2244 & -0.2995 & +0.2889 \\
\hline$\gamma_{a r}$ & -0.1927 & -0.2251 & -0.2483 & -0.2412 & -0.1699 & -0.1871 & -0.2227 & -0.1654 \\
\hline$K_{v}$ & +0.8296 & +0.8494 & +0.8910 & +0.8885 & +0.8984 & +0.9012 & +0.8973 & +0.8894 \\
\hline$\eta$ & +0.011 & +0.0564 & +0.0819 & +0.0230 & +0.0055 & +0.0642 & +0.0916 & +0.0420 \\
\hline
\end{tabular}

Table 8: PRCC values for the temperature- and rainfall dependent parameters of the model (2.1), using using total number of newly infectious humans as output with temperature values $T \in[16-20]^{\circ} \mathrm{C}$ and rainfall $R \in[60-120] \mathrm{mm}$. The top (most dominant) parameters that affect the dynamics of the model with respect to each of the four temperature ranges are highlighted in bold font. 